\title{
29. GEOCHEMICAL AND PETROLOGICAL CONSTRAINTS ON VELOCITY BEHAVIOR OF LOWER CRUSTAL AND UPPER MANTLE ROCKS FROM THE FAST-SPREADING RIDGE AT HESS DEEP ${ }^{1}$
}

\author{
D. Jay Miller, ${ }^{2}$ Gerardo J. Iturrino, ${ }^{3}$ and Nikolas I. Christensen ${ }^{4}$
}

\begin{abstract}
We present a multidisciplinary approach to investigating the physical properties of rocks interpreted to have been exhumed from the lower oceanic crust and upper mantle. Laboratory-measured compressional-wave velocities have been examined with respect to geochemical and petrological characteristics to help explain the seismic velocity structure of the oceanic lithosphere beneath the fast-spreading ridge near Hess Deep. Samples analyzed indicate that no bulk-rock chemical indices exhibit apparent correlation with velocity behavior. Differences between mean atomic weight predicted from velocity-density systematics and calculated from bulk-rock geochemistry can be ascribed to alteration effects and preferred mineral orientation. The primary controls on velocity behavior are similar in the gabbroic rocks sampled at Site 894 and the ultramafic rocks sampled at Site 895. In the gabbroic rocks, velocity behavior is controlled by alteration of clinopyroxene and possibly preferred mineral orientation. In the serpentinized peridotites, the primary control on velocity behavior is intensity of serpentinization. Comparison of velocity-depth profiles and mineral composition profiles highlights compositional trends that are not obvious from compositional data alone. Similar-scale, high-velocity gradients in cores from the fast-spreading ridge near Hess Deep, and the slow-spreading Southwest Indian Ridge, suggest that similar scale structural controls (on $\sim 150 \mathrm{~m}$ wavelength) may be present in both environments.
\end{abstract}

\section{INTRODUCTION}

Leg 147 of the Ocean Drilling Program (ODP) represents our first attempt at sampling the transition between the lower oceanic crust and upper mantle using an offset drilling strategy. Recognizing that drilling a complete section through the ocean crust is technologically challenging, this strategy of creating a composite section by drilling through tectonic windows where deep crustal and upper mantle rocks have been exhumed and emplaced at the seafloor represents a viable alternative sampling method. Although we acknowledge that exhumation, emplacement, and post-emplacement processes cloud our view of in situ conditions, attempts at peering through this veil are likely to be our only resource for providing constraints for the interpretation of seismic data until a continuous section through the oceanic crust is sampled intact.

Velocity behavior of these rocks is influenced by various parameters, including mineralogy, porosity, density, preferred mineral orientation, degree of alteration, and chemical composition. We have investigated the relative contribution of these parameters to velocity behavior to help constrain seismic data from the Hess Deep region. In another contribution to this volume, we examined velocity-porosity-density systematics and potential contributions of mineralogy and preferred mineral orientation to velocity behavior (Iturrino et al., this volume). In this paper, we explore correlations between chemical composition and compressional-wave velocity measured in representative samples of the various lithologies recovered from near Hess Deep (for site location map, see Iturrino et al., this volume).

Mével, C., Gillis, K.M., Allan, J.F., and Meyer, P.S. (Eds.), 1996. Proc. ODP, Sci. Results, 147: College Station, TX (Ocean Drilling Program).

${ }^{2}$ Ocean Drilling Program, Texas A\&M University Research Park, 1000 Discovery Drive, College Station, TX 77845, U.S.A. jaymiller@nelson.tamu.edu

${ }^{3}$ Rosenstiel School of Marine and Atmospheric Sciences, Division of Marine Geology and Geophysics, Miami, FL 33149, U.S.A.

${ }^{4}$ Department of Earth and Atmospheric Sciences, Purdue University, West Lafayette, IN 47907, U.S.A.
Examined in stratigraphic context, these data reveal a striking contrast in the velocity behavior of gabbroic rocks interpreted to represent a portion of the lower oceanic crust (Site 894) as compared to serpentinized peridotites believed to have been exhumed from the upper mantle (Site 895 ). Velocity-density relations, however, exhibit a robust correlation for both lithologic types, and intensity of alteration appears to be the primary control on rocks from both sites. We also examine, for comparison, the compressional-wave velocity data from the most complete section of lower oceanic crustal gabbroic rocks sampled to date: those from ODP Hole 735B at the Atlantis II Fracture Zone in the Southwest Indian Ocean.

\section{EXPERIMENTAL METHODS}

Leg 147 drilled one deep hole in the gabbroic section at Site 894, recovering $45 \mathrm{~m}$ of rock with a penetration of nearly $128 \mathrm{~m}$. In the peridotite section at Site 895 , two primary holes were drilled, both to a depth of $~ 90 \mathrm{~m}$ below seafloor (mbsf), with a total of $50 \mathrm{~m}$ of core recovered. The sampling strategy for this study was specifically designed to characterize the primary lithologic units recovered. During this research, 104 samples were examined: 44 samples from the gabbroic section and 60 from the peridotite section. Stratigraphic locations and a detailed description of velocity data acquisition are presented in Iturrino et al. (this volume). Whole-rock major element oxide analyses were performed by inductively coupled plasma analysis at Acme Analytical Laboratories Ltd., Vancouver, British Columbia, and the results are presented in Tables 1 and 2. Standard geochemical powders were included in the sample set as unknowns to ensure data quality. Mineral chemistry analyses were acquired from the Texas A\&M University Cameca SX-50 Superprobe. Natural and synthetic mineral standards were used for calibration. For space conservation, only average compositions for mineral phases are presented in this contribution (Tables 3,4). These average compositions represent more than 700 analyses, with at least five analyses per sample and multiple core-to-rim transects across minerals to establish intragrain heterogeneity. Complete analytical data tables are available from the authors. 


\section{PHYSICAL PROPERTIES BEHAVIOR OVERVIEW}

To evaluate the relative contribution of chemical composition to velocity behavior, we must first briefly examine other contributions. Iturrino et al. (this volume) provide an amplified discussion of physical properties behavior, and only the highlights of this analysis will be presented here. To minimize complications due to microcrack porosity, only velocity data at $200 \mathrm{MPa}$ is considered in this discussion. Density controls appear to vary according to rock type. In serpentinized dunites from the Hess Deep area, microcrack porosity can account for all the density variation observed (Iturrino et al., this volume). Although there is a distinct correlation between increasing porosity and decreasing density for serpentinized harzburgites from Site 895 , porosity increase alone is not sufficient to produce the observed density variation. Gabbroic rocks from the Hess Deep area are all generally of low porosity and exhibit no distinct porosity-density systematics, suggesting density in these rocks is controlled by mineralogic properties.

Despite the variable controls on density in the various rock types sampled from the Hess Deep area, there is a robust positive correlation between density and compressional-wave velocity $\left(V_{p}\right)$ for all the samples measured from Hess Deep (Fig. 1). Distinct fields are apparent for data from serpentinized dunites (low density-low $V_{p}$ ), serpentinized harzburgites (low to intermediate density and $V_{p}$ ), and gabbroic rocks (higher density and $V_{p}$ ), with some overlap between data sets. Three samples of basalt from a single unit in the gabbroic section have identical density and $V_{p}$. These basalts are holocrystalline and quite fresh. Therefore, they have densities and compressional-wave velocities similar to those of the gabbroic samples. A single basalt from the peridotite section has anomalously high $V_{p}$ and density. This sample is a pervasively amphibolitized, diabasic-textured basalt, and its physical properties are consistent with an amphibolite composition.

Birch $(1960,1961)$ documents the relationship between $V_{p}$ and density as it relates to chemistry in terms of mean atomic weight (MAW) in an attempt to overcome the individual effects of the various chemical components. Gabbro compositions compiled by Birch (1961) have a MAW of 21.8. According to lines of MAW corrected to $2 \mathrm{~kb}$ (Iturrino et al., 1991), gabbroic rocks from the Hess Deep area have comparable values of 20.5 to 22 , with an average of about 21.3 . In contrast, dunites (excluding Fe-rich compositions) in Birch's compilation (1961) have a MAW of 21. The serpentinized peridotites from Site 895 have significantly higher apparent mean atomic weights ( 21.7 to 22.5 ), interpreted by Iturrino et al. (this volume) to be representative of the pervasive alteration present in these rocks.

Finally, if we consider the compressional-wave velocity behavior of rocks sampled from Sites 894 and 895 independently, there is a marked difference in $V_{p}$ vs. depth profiles for the two sites. Figure 2 shows $V_{p}$ plotted in stratigraphic sequence for samples recovered from Site 894. These data define a trend of increasing $V_{p}$ with depth. Deviations from this trend in analyses 147-894G-6R, 10R, and 18R are due to pervasively altered samples. The strong positive inflection in velocity data from Section 147-894G-9R-4 corresponds to a change in lithology from gabbronorite to olivine gabbronorite. In contrast, $V_{p}$ data from the serpentinized peridotites sampled at Site 895 show no discernable trend with depth (Fig. 3).

\section{GEOCHEMICAL CONTRIBUTIONS TO VELOCITY BEHAVIOR}

\section{Whole-rock Geochemistry}

Iturrino et al. (this volume) present evidence that there are multiple controls on compressional-wave velocity behavior in rocks recovered from the Hess Deep area but that microcrack porosity, density, and preferred mineral orientation do not adequately account for all the observed variability in physical properties. Additionally, pet-

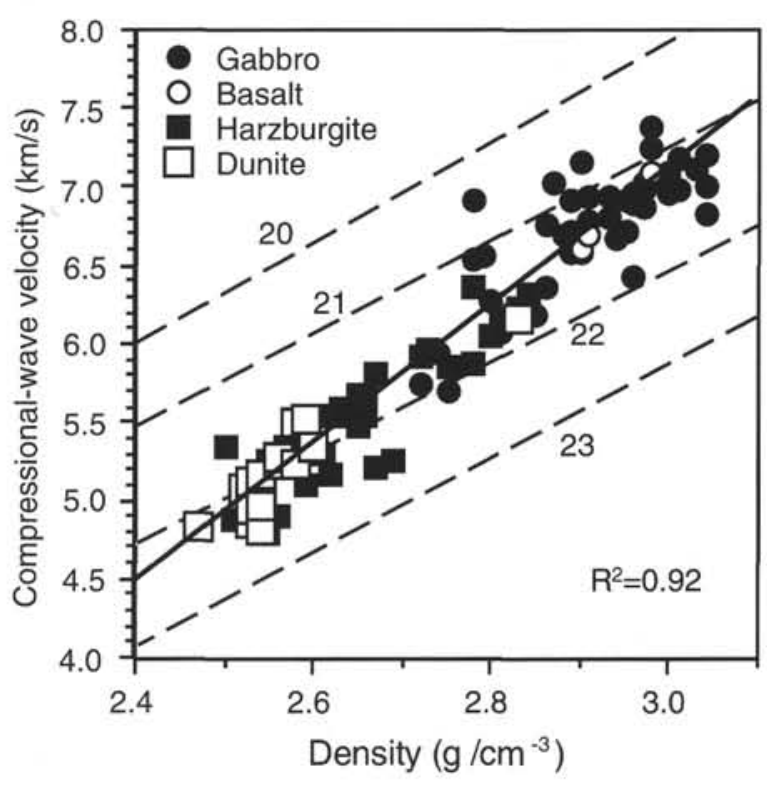

Figure 1. Velocity-density data for samples from Leg 147 distinguished by lithology. Superimposed dashed lines are from Birch (1961) as lines of constant mean atomic weight, corrected to $200 \mathrm{MPa}$ according to Iturrino et al. (1991). Solid line is calculated best-fit to all data.

rographic analysis indicates that no void space is present in any of the samples other than microcracks, so pore pressure is not a major contributor to velocity behavior. Of the primary contributors to velocity behavior that we can test with this sample set, only chemical composition and degree of alteration remain as potential controls. Tables 1 and 2 present the whole-rock major element oxide data acquired for samples from Sites 894 and 895 , respectively. Samples were selected as a subset of the minicores extracted for physical properties measurements and are representative of the major lithologies recovered.

Data in Table 1 are listed according to increasing $V_{p}$ to illustrate the lack of dependency of compressional-wave velocity on any whole-rock chemical index. No major element oxide or modal component exhibits any consistent behavior relative to $V_{p}$. Similarly, no modal component, or combination of modal components, appears to exert any control on velocity behavior. Common chemical indices of

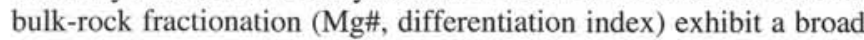
range of compositions correlative to any given $V_{p}$. Although there is broad range in $V_{p}$ for the gabbroic rocks recovered from near Hess Deep and a distinct variability in the chemical composition of these rocks, no correlations between these data sets are evident. Inasmuch as mineralogical variations are present (Table 1) but bulk-rock chemical compositions are similar (nearly all compositions are olivine and hypersthene normative; Table 1), variations in $V_{p}$ must reflect differences in degree of alteration or possibly mineral composition. Serpentinized peridotite compositions (Table 2) exhibit only minor compositional variability. This variability is expressed in minor fluctuations in the relative abundance of iron and magnesium.

Mean atomic weight calculated from bulk-rock analyses for gabbroic rocks compares favorably with estimates derived from densityvelocity relations, although the range (21.7 to 22.3 ) is more restricted and the average (22) is somewhat higher. Assuming Birch's lines of MAW are accurate, the data from gabbroic rocks in Figure 1 exhibit either anomalously low densities, or the velocities are much too high given the chemical compositions of the samples.

Mean atomic weights calculated from bulk-rock analyses for serpentinized peridotites are also different than those predicted from velocity-density relations. These calculated values range from 20.5 to 21.5 , with an average of 21 , suggesting that velocities in these ultra- 
Recovery

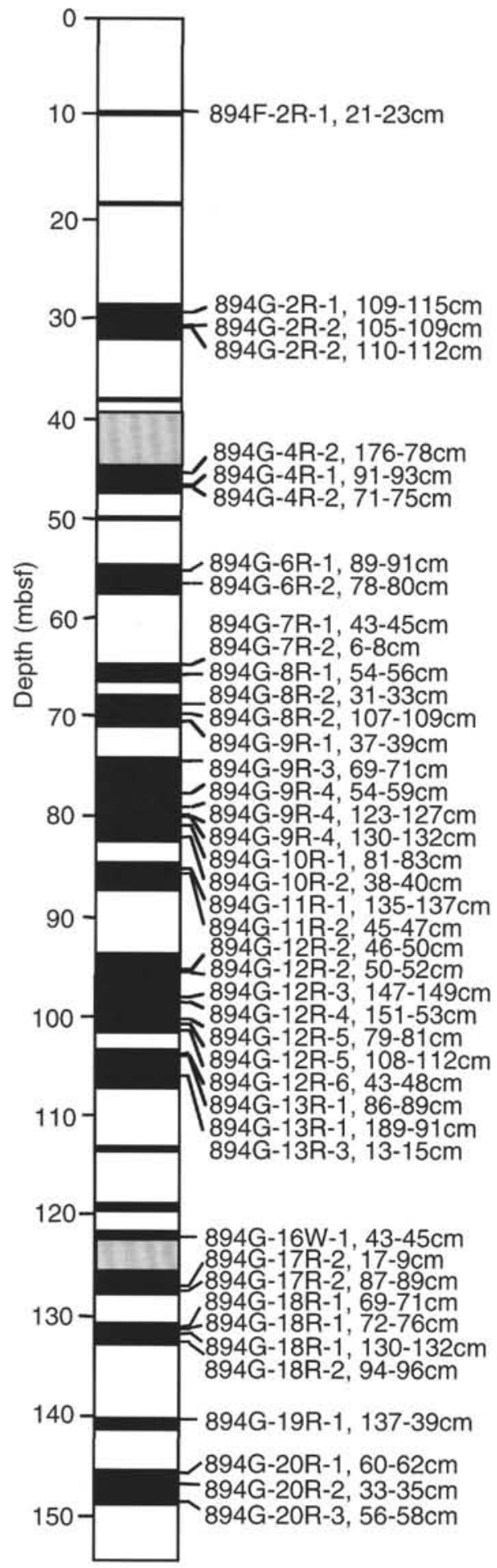

Holes 894F, G

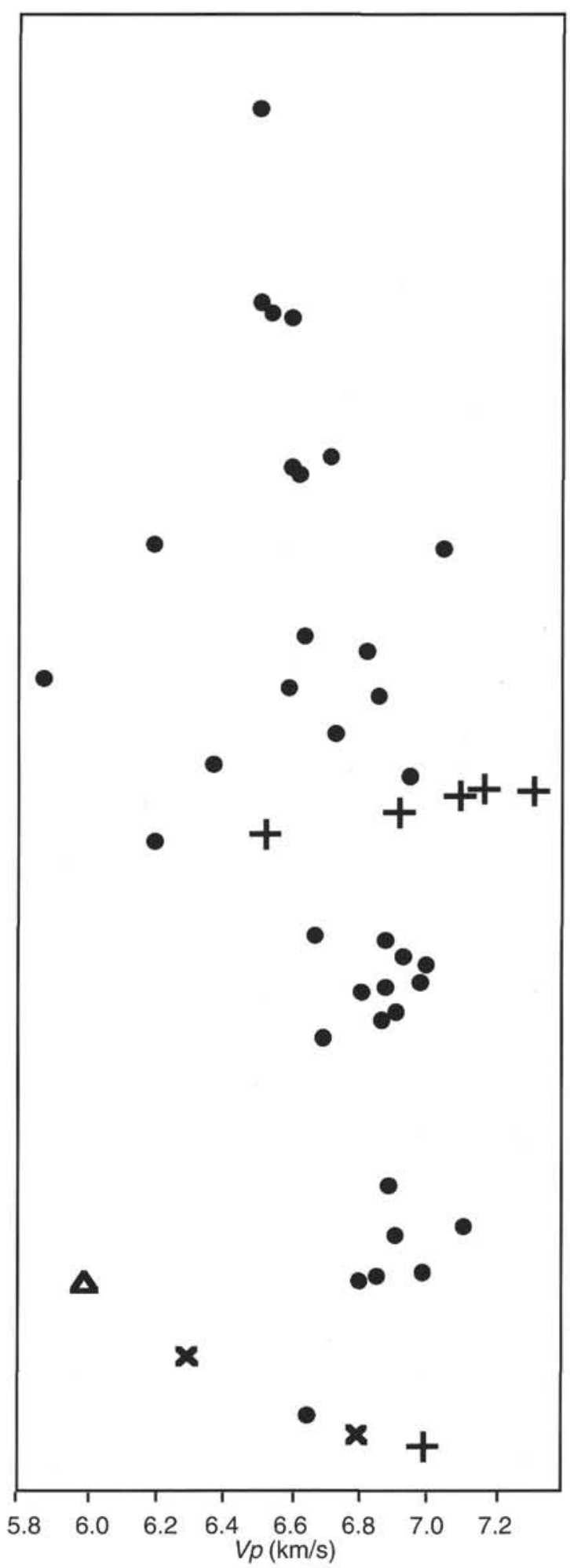

Figure 2. Velocity data plotted vs. depth for rocks from Site 894. Recovery is plotted in left column, represented by black bars. Sample locations within recovered intervals are noted. Gray-shaded areas in recovery column are intervals drilled but not cored. Symbols on right diagram: solid circle = gabbronorite; plus sign $=$ olivine gabbronorite $; \mathrm{x}=$ gabbro triangle $=$ cataclasite . 

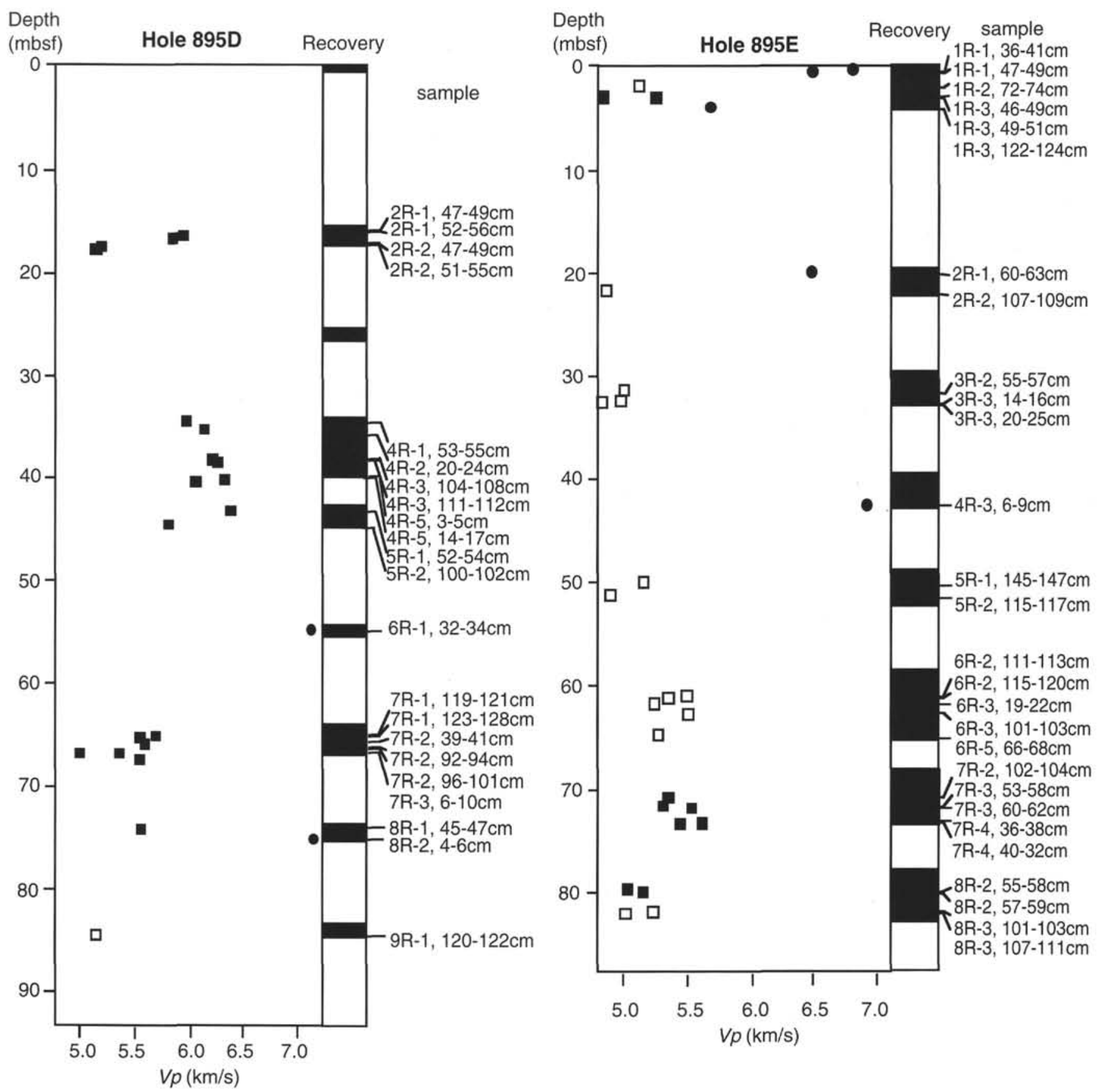

Figure 3. Velocity data plotted vs. depth for rocks from Site 895. Recovery is plotted in thin columns, represented by black bars. Sample locations within recovered intervals are noted. Symbols: solid square $=$ serpentinized harzburgite; open square $=$ serpentinized dunite; circle $=$ gabbroic rock .

mafic rocks are significantly lower (as much as $1 \mathrm{~km} / \mathrm{s}$ ) than would be predicted based on chemical composition. The reasons for the discrepancies between MAW derived from these alternative methods will be discussed below.

\section{Mineral Chemistry}

Clinopyroxene and plagioclase compositions were measured in 24 samples representing the various mafic lithologies recovered from Site 894 . Orthopyroxene and olivine compositions also were measured in many of these samples; however, there is little or no variability in the compositions of these phases. Therefore, variability in $V_{p}$ cannot be ascribed to compositional variability in these phases. Pol- ished thin sections were prepared from the same samples that were used to measure compressional-wave velocity, by slicing the end from a minicore after velocity measurements were completed and before the sample was pulverized for bulk chemical analysis. Averaged mineral chemistry data for each sample are compiled in Tables 3 and 4.

On first inspection, there appears to be minor (or no) depth-related compositional variability in either clinopyroxene $\mathrm{Mg \#}$ or the anorthite composition of plagioclase (Fig, 4). When we examine these data in light of the sharp positive inflection in velocity data present in samples from Section 147-894G-9R-4 from a depth of $80 \mathrm{mbsf}$, we recognize a distinct break in the compositional profile for clinopyroxene, which thus subdivided, exhibits a more explicit downhole trend (Fig. 5). Pyroxene compositions in samples from Hole $894 \mathrm{G}$ become 
Table 1. Whole-rock geochemistry, normative mineralogy, geochemical indices of fractionation, and mineral modes for gabboric rocks from Site 894.

\begin{tabular}{|c|c|c|c|c|c|c|c|c|c|c|c|c|c|c|c|c|}
\hline Hole: & $894 \mathrm{G}$ & $894 \mathrm{G}$ & $894 \mathrm{G}$ & $894 \mathrm{~F}$ & $894 \mathrm{G}$ & $894 \mathrm{G}$ & $894 \mathrm{G}$ & $894 \mathrm{G}$ & $894 \mathrm{G}$ & $894 \mathrm{G}$ & $894 \mathrm{G}$ & $894 \mathrm{G}$ & $894 \mathrm{G}$ & $894 \mathrm{G}$ & $894 \mathrm{G}$ & $894 \mathrm{G}$ \\
\hline Core, section: & $11 \mathrm{R}-2$ & $19 \mathrm{R}-1$ & $9 \mathrm{R}-3$ & $2 \mathrm{R}-1$ & $2 \mathrm{R}-1$ & $2 \mathrm{R}-2$ & $7 \mathrm{R}-1$ & $20 \mathrm{R}-1$ & $12 \mathrm{R}-2$ & $4 \mathrm{R}-1$ & $13 R-3$ & $12 \mathrm{R}-6$ & $18 \mathrm{R}-1$ & $13 R-1$ & $6 \mathrm{R}-2$ & $9 \mathrm{R}-4$ \\
\hline Interval: & $45-47$ & $37-39$ & $69-71$ & $21-23$ & $109-115$ & $105-109$ & $43-45$ & $60-62$ & $46-50$ & $91-93$ & $13-15$ & $43-48$ & $72-76$ & 89-91 & $78-80$ & $123-127$ \\
\hline \multicolumn{17}{|l|}{ Component (wt\%) } \\
\hline $\mathrm{SiO}_{2}$ & 51.16 & 49.11 & 49.22 & 50.87 & 47.79 & 47.56 & 50.17 & 51.31 & 51.69 & 51.54 & 51.67 & 50.17 & 50.66 & 50.09 & 51.61 & 47.60 \\
\hline $\mathrm{TiO}_{2}$ & 0.47 & 0.38 & 0.60 & 0.85 & 0.95 & 0.90 & 0.67 & 0.43 & 0.46 & 0.89 & 0.85 & 0.71 & 0.58 & 0.63 & 0.81 & 0.38 \\
\hline $\mathrm{Al}_{2} \mathrm{O}_{3}$ & 15.69 & 16.97 & 17.03 & 14.93 & 17.68 & 18.41 & 14.51 & 16.59 & 17.77 & 14.79 & 14.52 & 13.91 & 20.72 & 13.97 & 13.86 & 16.33 \\
\hline $\mathrm{Fe}_{2} \mathrm{O}_{3}$ & 7.88 & 8.06 & 8.48 & 10.21 & 8.05 & 7.59 & 10.00 & 7.18 & 6.93 & 7.63 & 9.00 & 10.46 & 5.68 & 9.64 & 7.66 & 9.17 \\
\hline $\mathrm{MgO}$ & 9.42 & 8.67 & 9.76 & 7.96 & 9.57 & 9.23 & 9.50 & 9.10 & 7.64 & 8.24 & 9.22 & 8.43 & 5.56 & 9.26 & 9.63 & 11.84 \\
\hline $\mathrm{MnO}$ & 0.16 & 0.14 & 0.15 & 0.17 & 0.15 & 0.14 & 0.15 & 0.15 & 0.14 & 0.16 & 0.17 & 0.18 & 0.10 & 0.17 & 0.16 & 0.14 \\
\hline $\mathrm{CaO}$ & 12.81 & 11.82 & 11.78 & 11.33 & 11.92 & 11.98 & 11.41 & 12.30 & 11.14 & 14.31 & 11.21 & 12.72 & 12.79 & 13.41 & 13.29 & 11.21 \\
\hline $\mathrm{Na}_{2} \mathrm{O}$ & 2.15 & 2.89 & 2.20 & 2.85 & 2.11 & 2.00 & 2.49 & 2.25 & 2.95 & 2.23 & 2.46 & 2.51 & 3.33 & 2.31 & 2.22 & 2.14 \\
\hline $\mathrm{K}_{2} \mathrm{O}$ & 0.06 & 0.11 & 0.12 & 0.26 & 0.05 & 0.05 & 0.26 & 0.07 & 0.08 & 0.05 & 0.06 & & 0.12 & 0.08 & 0.06 & 0.19 \\
\hline $\mathrm{P}_{2} \mathrm{O}_{5}$ & 0.02 & 0.02 & 0.04 & 0.06 & 0.07 & 0.06 & 0.07 & 0.02 & 0.03 & 0.06 & 0.04 & 0.02 & 0.03 & 0.02 & 0.08 & 0.04 \\
\hline LOI & 0.4 & 1.8 & 0.7 & 0.3 & 1.5 & 1.8 & 0.7 & 0.5 & 0.9 & 0.4 & 0.6 & 0.4 & 0.5 & 0.4 & 0.52 & 1.1 \\
\hline Total & 100.26 & 100.02 & 99.97 & 99.81 & 99.86 & 99.73 & 99.97 & 99.94 & 99.76 & 100.32 & 100.02 & 99.54 & 100.11 & 100.02 & 99.93 & 100.20 \\
\hline $2 \mathrm{~kb} V_{p}$ & 6.18 & 6.36 & 6.43 & 6.58 & 6.58 & 6.60 & 6.71 & 6.71 & 6.76 & 6.78 & 6.83 & 6.88 & 6.91 & 6.96 & 7.11 & 7.24 \\
\hline \multicolumn{17}{|l|}{ Normative (\%) } \\
\hline Orthoclase & 0.35 & 0.65 & 0.71 & 1.54 & 0.30 & 0.30 & 1.54 & 0.41 & 0.47 & 0.30 & 0.35 & & 0.71 & 0.47 & 0.35 & 1.12 \\
\hline Albite & 18.19 & 24.45 & 18.62 & 24.12 & 17.85 & 16.92 & 21.07 & 19.04 & 24.96 & 18.87 & 20.82 & 21.24 & 28.18 & 19.55 & 18.78 & 18.11 \\
\hline Anorthite & 32.98 & 33.01 & 36.42 & 27.18 & 38.62 & 41.11 & 27.65 & 34.96 & 35.01 & 30.20 & 28.40 & 26.69 & 41.23 & 27.51 & 27.69 & 34.39 \\
\hline Diopside & 24.57 & 20.65 & 17.72 & 23.39 & 16.16 & 14.44 & 23.15 & 20.90 & 16.23 & 32.65 & 21.82 & 29.62 & 17.85 & 31.58 & 30.38 & 16.90 \\
\hline Hypersthene & 17.64 & 1.47 & 12.21 & 12.30 & 9.82 & 11.28 & 12.52 & 19,70 & 17.42 & 14.21 & 23.95 & 10.53 & 0.37 & 7.47 & 17.58 & 3.98 \\
\hline Olivine & 3.29 & 15.27 & 10.66 & 6.82 & 11.74 & 10.24 & 9.53 & 1.84 & 2.17 & 0.07 & & 7.18 & 8.72 & 9.50 & 1.08 & 21.57 \\
\hline Magnetite & 1.14 & 1.17 & 1.23 & 1.48 & 1.17 & 1.10 & 1.45 & 1.04 & 1.01 & 1.11 & 1.31 & 1.52 & 0.82 & 1.40 & 1.11 & 1.33 \\
\hline Ilmenite & 0.89 & 0.72 & 1.14 & 1.61 & 1.80 & 1.71 & 1.27 & 0.82 & 0.87 & $\begin{array}{l}1.69 \\
\end{array}$ & 1.61 & 1.35 & 1.10 & 1.20 & 1.54 & 0.72 \\
\hline Apatite & 0.04 & 0.04 & 0.09 & 0.13 & 0.15 & 0.13 & 0.15 & 0.04 & 0.07 & 0.13 & 0.09 & 0.04 & 0.07 & 0.04 & 0.17 & 0.09 \\
\hline \multicolumn{17}{|c|}{ Fractionation indices } \\
\hline Mg\# & 72.5 & 70.3 & 71.8 & 63.2 & 72.4 & 72.8 & 67.6 & 73.6 & 70.8 & 70.4 & 69.3 & 64.0 & 68.3 & 67.9 & 73.5 & 74.0 \\
\hline Total alkalis & 2.21 & 3.00 & 2.32 & 3.11 & 2.16 & 2.05 & 2.75 & 2.32 & 3.03 & 2.28 & 2.52 & 2.51 & 3.45 & 2.39 & 2.29 & 2.33 \\
\hline D.I. & 18.5 & 25.1 & 19.3 & 25.7 & 18.5 & 17.2 & 22.6 & 19.5 & 25.4 & 19.2 & 21.2 & 21.2 & 28.9 & 20.0 & 19.2 & 19.2 \\
\hline \multicolumn{17}{|l|}{ Mode $(\%)$} \\
\hline Plagioclase & 50 & 60 & 45 & 51 & Basalt & Basalt & 60 & 45 & 60 & 55 & 55 & 50 & 60 & 53 & 45 & 55 \\
\hline Clinopyroxene & 40 & 25 & 45 & 47 & Dastant & Dosatr & 20 & 25 & 25 & 30 & 30 & 35 & 35 & 30 & 35 & 35 \\
\hline Orthopyroxene & 10 & 15 & & & & & 15 & 30 & 15 & 15 & 13 & 15 & 5 & 15 & 25 & 5 \\
\hline $\begin{array}{l}\text { Olivine } \\
\text { Oxides }\end{array}$ & Trace & Trace & 10 & 2 & & & Trace & Trace & Trace & Trace & 2 & Trace & Trace & 2 & Trace & Trace \\
\hline
\end{tabular}

Notes: $\mathrm{Mg} \#=\mathrm{Mg} /(\mathrm{Mg}+\mathrm{Fe})$; D.L. is Thornton and Tuttle's (1960) differentiation index. 
Table 2. Whole-rock geochemistry and percent total alteration for ultramafic rocks from Site 895 .

\begin{tabular}{|c|c|c|c|c|c|c|c|c|}
\hline Hole: & $895 \mathrm{C}$ & $895 \mathrm{C}$ & $895 \mathrm{D}$ & $895 \mathrm{D}$ & $895 \mathrm{E}$ & $895 \mathrm{E}$ & $895 \mathrm{E}$ & $895 \mathrm{E}$ \\
\hline Core, section: & $3 R-2$ & $4 \mathrm{R}-2$ & $4 \mathrm{R}-2$ & $7 R-2$ & $3 R-3$ & $6 R-2$ & $6 \mathrm{R}-3$ & $7 R-4$ \\
\hline Interval: & $30-34$ & $17-20$ & $20-24$ & $96-99$ & $14-16$ & $115-120$ & $19-22$ & $40-43$ \\
\hline \multicolumn{9}{|l|}{ Component (wt $\%$ ) } \\
\hline $\mathrm{SiO}_{2}$ & 35.31 & 34.44 & 36.98 & 35.05 & 32.71 & 33.33 & 32.70 & 35.58 \\
\hline $\mathrm{TiO}_{2}$ & 0.03 & 0.07 & 0.02 & 0.03 & 0.02 & 0.03 & 0.03 & 0.02 \\
\hline $\mathrm{Al}_{2} \mathrm{O}_{3}$ & 0.80 & 3.67 & 0.53 & 0.88 & 0.31 & 0.25 & 0.23 & 0.49 \\
\hline $\mathrm{Fe}_{2} \mathrm{O}_{3}$ & 8.20 & 10.07 & 8.84 & 7.87 & 10.14 & 8.29 & 8.67 & 8.46 \\
\hline $\mathrm{MgO}^{\circ}$ & 41.77 & 39.81 & 43.24 & 41.36 & 41.42 & 42.98 & 42.54 & 41.92 \\
\hline $\mathrm{MnO}$ & 0.12 & 0.14 & 0.12 & 0.11 & 0.14 & 0.11 & 0.12 & 0.11 \\
\hline $\mathrm{CaO}$ & 0.50 & 1.53 & 0.64 & 0.22 & 0.06 & 0.11 & 0.07 & 0.41 \\
\hline $\mathrm{Na}_{2} \mathrm{O}$ & 0.05 & 0.21 & 0.01 & 0.03 & & & & \\
\hline $\mathrm{K}_{2} \mathrm{O}$ & 0.06 & 0.15 & 0.15 & 0.09 & 0.14 & 0.15 & & \\
\hline $\mathrm{P}_{2} \mathrm{O}_{5}$ & 0.08 & 0.07 & 0.04 & 0.07 & 0.07 & 0.05 & 0.06 & 0.06 \\
\hline LOI & 12.50 & 10.30 & 8.90 & 14.00 & 14.70 & 14.60 & 15.00 & 12.60 \\
\hline Total & 99.88 & 100.50 & 99.91 & 100.02 & 99.89 & 99.97 & 99.81 & 100.12 \\
\hline \multirow[t]{2}{*}{$2 \mathrm{~kb} V_{p}$} & 5.33 & 5.73 & 6.13 & 5.00 & 4.85 & 5.28 & 5.23 & 5.46 \\
\hline & $\mathrm{H}$ & pl-D & pl-D & $\mathrm{H}$ & $\mathrm{D}$ & D & D & $\mathrm{H}$ \\
\hline Alteration (\%) & 56 & 55 & 79 & 100 & 99 & 90 & 94 & 85 \\
\hline
\end{tabular}

Notes: $\mathrm{H}=$ harzburgite; $\mathrm{D}=$ dunite; $\mathrm{pl}-\mathrm{D}=$ impregnated dunite.

less magnesian from the bottom of the core to an approximate depth of 95 mbsf (Section 147-894G-12R-2). Above this section there is an abrupt change to more primitive olivine gabbronorite (Cores 147$894 \mathrm{G}-9 \mathrm{R}$ and 10R), which is overlain by a sequence of gabbronorite and gabbro. Over this same transition, pyroxene composition exhibits an abrupt increase in magnesium content, and above $95 \mathrm{mbsf}$ the profile again exhibits a steady decay.

Plagioclase composition over this same interval is less indicative of an evolutionary trend because of the large range in compositions displayed in most samples. While there is no apparent change in plagioclase composition in samples from the bottom of Hole $894 \mathrm{G}$ to a depth of 95 mbsf, above this point there is an indication of decreasing anorthite content, consistent with the decreasing clinopyroxene $\mathrm{Mg \#}$. However, this trend may be biased by the composition of the uppermost sample, in which case, there may be no compositional trend reflected in plagioclase composition.

\section{DISCUSSION}

This project was conceived based on success in investigating geochemical constraints on laboratory-measured compressionalwave velocities from other environments (e.g., Miller and Christensen, 1994). During this investigation, five primary questions regarding velocity behavior were identified: (1) Does bulk-rock chemistry, which reflects the diversity in lithologies recovered during Leg 147, demonstrate any correlative properties that can be used to interpret velocity behavior in the lower oceanic crust and upper mantle? (2) What causes the difference between MAW predicted from densityvelocity systematics and calculated from bulk-rock chemical analyses? (3) Can we identify the primary controls on velocity behavior, and are they the same in gabbroic rocks and peridotites? (4) What does correlation between $V_{p}$-depth and mineral chemistry-depth profiles tell us about the nature of the lower oceanic crust? (5) Although the depth of penetration and sampling on Leg 147 was limited, can the data we have generated be related to other environments where the lower oceanic crust has been sampled, and how does this data influence our construction of a composite stratigraphic section?

Inasmuch as this study was intended to examine background variability for potential correlation with remote-sensing models, it was no surprise that no correlations were apparent between the bulk-rock chemical composition and velocity behavior of serpentinized peridotites sampled from Site 895. Samples of local, small-scale features were intentionally not included in this sample suite, as these might have biased our observations toward anomalies that would be beyond the resolution of remote-sensing techniques. Hence, shipboard petro- graphic analyses demonstrated all these samples were pervasively serpentinized (Gillis, Mével, Allan, et al., 1993), and shipboard and shore-based bulk-rock geochemical analyses were remarkably homogeneous (Mg\# 89-91) despite a broad range $(5 \mathrm{~km} / \mathrm{s}$ to $6.5 \mathrm{~km} / \mathrm{s})$ in laboratory-measured compressional-wave velocities. Because samples representative of principal lithologies were analyzed, we surmise that either there is no recognizable geochemical contribution to compressional-wave velocity behavior in the upper mantle rocks from the Hess Deep region or that any geochemical contribution has been overprinted by processes of exhumation, hydration, and emplacement at the seafloor.

Gabbroic rocks sampled from Site 894, however, exhibit a range of chemical compositions as well as $V_{p}$ values. To reduce the potential that alteration effects would contribute to velocity variability, only the least altered cores were selected for our analyses. Despite the potential for correlative parameters, as with the serpentinized peridotites from Site 895 , we cannot distinguish a distinct control on velocity behavior attributable to bulk-rock chemistry. We ascribe this lack of recognizable control to several possibilities. First, the variations in lithology and bulk-rock chemistry that we have identified are due to variations in the ratios of the primary mineral phases present. Clinopyroxene-orthopyroxene ratios, while having a significant effect on bulk-chemistry, are not likely to generate much difference in laboratory-measured compressional-wave velocities in mineral aggregates where these phases comprise less than half the rock. Plagioclase modal content is, in our sample suite, relatively invariant. Ideal compressional-wave velocities for mineral aggregates with the ratios of principal phases exhibited by our sample suite have a range of 6.9 to $7.2 \mathrm{~km} / \mathrm{s}$ (from Voigt-Reuss-Hill averages [VRH]; Hill, 1952), governed by the amount of olivine in the aggregate. Since olivine content will also have the most significant effect on iron-magnesium ratios in these rocks, we should be able to recognize compositional contributions to velocity behavior. Indeed, our most primitive composition sample (147-894G-9R-4, 123-127 cm) does exhibit the highest compressional-wave velocity measured in this suite.

Second, normative calculations based on bulk-chemical analyses for gabbroic rocks from Site 894 suggest that velocity differences notwithstanding, our sample suite is too compositionally homogeneous to recognize correlative trends (Table 1). There is an indication in the samples with the four highest $V_{p}$ values (Table 1), which fall within the ideal VRH velocities window, of a fractionation trend. In these samples we see that progressively lower velocities are associated with progressively more evolved chemical compositions. However, this trend is not supported by the balance of our data.

Finally, even though we selected the least altered gabbroic samples available for this synthesis, all were minimally $20 \%$ altered. In- 
Table 3. Average clinopyroxene compositions from gabbroic rocks sampled at Site 894 .

\begin{tabular}{|c|c|c|c|c|c|c|c|c|c|c|c|}
\hline Hole: & $894 \mathrm{~F}$ & $894 \mathrm{G}$ & $894 G$ & $894 G$ & $894 G$ & $894 \mathrm{G}$ & $894 \mathrm{G}$ & $894 \mathrm{G}$ & $894 G$ & $895 \mathrm{G}$ & $894 \mathrm{G}$ \\
\hline Core, section: & $2 \mathrm{R}-1$ & $4 \mathrm{R}-1$ & 6R-2 & $7 R-1$ & $8 \mathrm{R}-2$ & $9 \mathrm{R}-1$ & $9 R-4$ & $10 \mathrm{R}-1$ & $10 \mathrm{R}-2$ & $11 \mathrm{R}-1$ & $12 \mathrm{R}-2$ \\
\hline Interval: & $21-23$ & $91-93$ & $82-85$ & $43-45$ & $107-109$ & $37-39$ & $54-59$ & $85-87$ & $38-40$ & $135-137$ & $46-50$ \\
\hline Depth (mbsf): & 9.53 & 45.91 & 57.05 & 65.25 & 71.08 & 74.77 & 78.85 & 79.95 & 80.86 & 85.47 & 95.76 \\
\hline \multicolumn{12}{|c|}{ Component (wt $\%$ ) } \\
\hline $\mathrm{Na}_{2} \mathrm{O}$ & 0.29 & 0.32 & 0.33 & 0.33 & 0.33 & 0.33 & 0.34 & 0.35 & 0.34 & 0.32 & 0.35 \\
\hline $\mathrm{SiO}_{2}$ & 51.18 & 50.67 & 50.91 & 50.69 & 50.79 & 51.26 & 50.89 & 50.98 & 50.76 & 50.77 & 50.98 \\
\hline $\mathrm{MgO}$ & 14.75 & 14.60 & 15.13 & 14.74 & 15.19 & 15.12 & 15.33 & 15.12 & 14.71 & 15.35 & 13.95 \\
\hline $\mathrm{Al}_{2} \mathrm{O}_{3}$ & 2.05 & 2.18 & 2.17 & 2.05 & 2.24 & 2.00 & 2.46 & 2.25 & 2.14 & 2.30 & 1.86 \\
\hline $\mathrm{K}_{2} \mathrm{O}$ & 0.01 & 0.00 & 0.01 & 0.01 & 0.01 & 0.01 & 0.01 & 0.01 & 0.01 & 0.00 & 0.02 \\
\hline $\mathrm{CaO}$ & 18.81 & 20.26 & 19.84 & 19.46 & 19.42 & 19.10 & 20.29 & 19.87 & 20.24 & 19.58 & 20.78 \\
\hline $\mathrm{Cr}_{2} \mathrm{O}_{3}$ & 0.04 & 0.12 & 0.10 & 0.11 & 0.17 & 0.08 & 0.35 & 0.17 & 0.15 & 0.26 & 0.03 \\
\hline $\mathrm{TiO}_{2}$ & 0.72 & 0.78 & 0.78 & 0.67 & 0.72 & 0.72 & 0.67 & 0.69 & 0.72 & 0.65 & 0.72 \\
\hline $\mathrm{FeO}^{2}$ & 9.78 & 7.51 & 7.32 & 8.21 & 7.61 & 8.68 & 6.21 & 7.26 & 7.11 & 7.00 & 7.79 \\
\hline $\mathrm{NiO}$ & 0.02 & 0.02 & 0.03 & 0.02 & 0.02 & 0.04 & 0.02 & 0.03 & 0.03 & 0.02 & 0.04 \\
\hline $\mathrm{MnO}$ & 0.27 & 0.26 & 0.26 & 0.25 & 0.25 & 0.27 & 0.21 & 0.22 & 0.28 & 0.25 & 0.30 \\
\hline $\mathrm{Fe}_{2} \mathrm{O}_{3}$ & 2.09 & 3.15 & 2.92 & 2.96 & 3.08 & 2.35 & 2.82 & 3.00 & 3.17 & 3.17 & 2.51 \\
\hline Total & 100.02 & 99.87 & 99.82 & 99.47 & 99.83 & 99.96 & 99.59 & 99.95 & 99.65 & 99.66 & 99.32 \\
\hline \multicolumn{12}{|l|}{ Ions per 60} \\
\hline $\mathrm{Na}$ & 0.02 & 0.02 & 0.02 & 0.02 & 0.02 & 0.02 & 0.02 & 0.03 & 0.02 & 0.02 & 0.03 \\
\hline $\mathrm{Si}$ & 1.92 & 1.90 & 1.90 & 1.90 & 1.90 & 1.91 & 1.89 & 1.90 & 1.90 & 1.90 & 1.92 \\
\hline $\mathrm{Mg}$ & 0.82 & 0.81 & 0.84 & 0.83 & 0.85 & 0.84 & 0.85 & 0.84 & 0.82 & 0.85 & 0.79 \\
\hline $\mathrm{Al}$ & 0.09 & 0.10 & 0.10 & 0.09 & 0.10 & 0.09 & 0.11 & 0.10 & 0.09 & 0.10 & 0.08 \\
\hline $\mathrm{K}$ & 0.00 & 0.00 & 0.00 & 0.00 & 0.00 & 0.00 & 0.00 & 0.00 & 0.00 & 0.00 & 0.00 \\
\hline $\mathrm{Ca}$ & 0.75 & 0.81 & 0.79 & 0.78 & 0.78 & 0.76 & 0.81 & 0.79 & 0.81 & 0.78 & 0.84 \\
\hline $\mathrm{Cr}$ & 0.00 & 0.00 & 0.00 & 0.00 & 0.01 & 0.00 & 0.01 & 0.01 & 0.00 & 0.01 & 0.00 \\
\hline $\mathrm{Ti}$ & 0.02 & 0.02 & 0.02 & 0.02 & 0.02 & 0.02 & 0.02 & 0.02 & 0.02 & 0.02 & 0.02 \\
\hline $\mathrm{Fe}^{2+}$ & 0.31 & 0.23 & 0.23 & 0.26 & 0.24 & 0.27 & 0.19 & 0.23 & 0.22 & 0.22 & 0.25 \\
\hline $\mathrm{Ni}$ & 0.00 & 0.00 & 0.00 & 0.00 & 0.00 & 0.00 & 0.00 & 0.00 & 0.00 & 0.00 & 0.00 \\
\hline $\mathrm{Mn}$ & 0.01 & 0.01 & 0.01 & 0.01 & 0.01 & 0.01 & 0.01 & 0.01 & 0.01 & 0.01 & 0.01 \\
\hline $\mathrm{Fe}^{3+}$ & 0.06 & 0.09 & 0.08 & 0.08 & 0.09 & 0.07 & 0.08 & 0.08 & 0.09 & 0.09 & 0.07 \\
\hline Total & 4.00 & 4.00 & 4.00 & 4.00 & 4.00 & 4.00 & 4.00 & 4.00 & 4.00 & 4.00 & 4.00 \\
\hline Mg\# & 0.73 & 0.78 & 0.79 & 0.76 & 0.78 & 0.76 & 0.82 & 0.79 & 0.79 & 0.80 & 0.76 \\
\hline
\end{tabular}

Table 3 (continued).

\begin{tabular}{|c|c|c|c|c|c|c|c|c|c|c|c|}
\hline Hole: & $894 \mathrm{G}$ & $894 \mathrm{G}$ & $894 \mathrm{G}$ & $894 \mathrm{G}$ & $894 \mathrm{G}$ & $894 \mathrm{G}$ & $894 \mathrm{G}$ & $894 \mathrm{G}$ & $894 \mathrm{G}$ & $894 \mathrm{G}$ & $894 G$ \\
\hline Core, section: & $12 \mathrm{R}-3$ & 13R-1 & $13 R-3$ & $15 \mathrm{R}-1$ & $17 R-1$ & $17 \mathrm{R}-2$ & $18 \mathrm{R}-1$ & 19R-1 & 20R-1 & 20R-1 & 20R-2 \\
\hline Interval: & $147-149$ & $89-91$ & $13-15$ & $77-80$ & $123-125$ & $87-89$ & $130-132$ & $37-39$ & $19-22$ & $56-59$ & $33-35$ \\
\hline Depth (mbsf): & 98.21 & 104.29 & 106.38 & 119.57 & 127.03 & 128.12 & 132.22 & 140.89 & 145.82 & 146.16 & 147.43 \\
\hline \multicolumn{12}{|c|}{ Component (wt\%) } \\
\hline $\mathrm{Na}_{2} \mathrm{O}$ & 0.33 & 0.32 & 0.32 & 0.29 & 0.27 & 0.32 & 0.33 & 0.33 & 0.31 & 0.34 & 0.33 \\
\hline $\mathrm{SiO}_{2}$ & 50.68 & 50.95 & 50.37 & 50.83 & 50.86 & 50.77 & 50.82 & 50.83 & 50.69 & 50.90 & 50.30 \\
\hline $\mathrm{MgO}$ & 14.68 & 14.88 & 14.85 & 14.99 & 15.28 & 15.04 & 15.53 & 14.69 & 14.91 & 14.99 & 14.96 \\
\hline $\mathrm{Al}_{2} \mathrm{O}_{3}$ & 2.05 & 2.06 & 2.02 & 2.02 & 1.92 & 2.09 & 2.19 & 2.33 & 2.13 & 2.50 & 2.20 \\
\hline $\mathrm{K}_{2} \mathrm{O}$ & 0.01 & 0.01 & 0.01 & 0.01 & 0.00 & 0.01 & 0.00 & 0.02 & 0.01 & 0.01 & 0.01 \\
\hline $\mathrm{CaO}$ & 20.15 & 19.71 & 19.37 & 19.61 & 19.21 & 19.83 & 19.24 & 20.46 & 20.10 & 20.74 & 20.06 \\
\hline $\mathrm{Cr}_{2} \mathrm{O}_{3}$ & 0.17 & 0.10 & 0.12 & 0.13 & 0.13 & 0.16 & 0.17 & 0.24 & 0.11 & 0.31 & 0.18 \\
\hline $\mathrm{TiO}_{2}$ & 0.74 & 0.66 & 0.75 & 0.84 & 0.71 & 0.82 & 0.76 & 0.71 & 0.86 & 0.62 & 0.78 \\
\hline $\mathrm{FeO}$ & 7.85 & 7.92 & 7.80 & 8.03 & 8.02 & 7.50 & 7.33 & 7.17 & 7.34 & 6.23 & 6.64 \\
\hline $\mathrm{NiO}$ & 0.01 & 0.02 & 0.04 & 0.03 & 0.02 & 0.01 & 0.01 & 0.02 & 0.01 & 0.03 & 0.02 \\
\hline $\mathrm{MnO}$ & 0.25 & 0.27 & 0.28 & 0.28 & 0.31 & 0.21 & 0.25 & 0.27 & 0.26 & 0.22 & 0.25 \\
\hline $\mathrm{Fe}_{2} \mathrm{O}_{3}$ & 2.62 & 3.04 & 3.51 & 3.13 & 3.21 & 3.23 & 3.11 & 2.97 & 2.94 & 2.84 & 3.53 \\
\hline Total & 99.55 & 99.95 & 99.43 & 100.18 & 99.93 & 99.99 & 99.74 & 100.01 & 99.66 & 99.75 & 99.27 \\
\hline \multicolumn{12}{|l|}{ Ions per 60} \\
\hline $\mathrm{Na}$ & 0.02 & 0.02 & 0.02 & 0.02 & 0.02 & 0.02 & 0.02 & 0.02 & 0.02 & 0.02 & 0.02 \\
\hline $\mathrm{Si}$ & 1.91 & 1.90 & 1.89 & 1.90 & 1.90 & 1.90 & 1.90 & 1.90 & 1.90 & 1.90 & 1.89 \\
\hline $\mathrm{Mg}$ & 0.82 & 0.83 & 0.83 & 0.83 & 0.85 & 0.84 & 0.86 & 0.82 & 0.83 & 0.83 & 0.84 \\
\hline $\mathrm{Al}$ & 0.09 & 0.09 & 0.09 & 0.09 & 0.08 & 0.09 & 0.10 & 0.10 & 0.09 & 0.11 & 0.10 \\
\hline $\mathrm{K}$ & 0.00 & 0.00 & 0.00 & 0.00 & 0.00 & 0.00 & 0.00 & 0.00 & 0.00 & 0.00 & 0.00 \\
\hline $\mathrm{Ca}$ & 0.80 & 0.79 & 0.78 & 0.78 & 0.77 & 0.79 & 0.77 & 0.82 & 0.81 & 0.83 & 0.81 \\
\hline $\mathrm{Cr}$ & 0.01 & 0.00 & 0.00 & 0.00 & 0.00 & 0.00 & 0.01 & 0.01 & 0.00 & 0.01 & 0.01 \\
\hline $\mathrm{Ti}$ & 0.02 & 0.02 & 0.02 & 0.02 & 0.02 & 0.02 & 0.02 & 0.02 & 0.02 & 0.02 & 0.02 \\
\hline $\mathrm{Fe}^{2+}$ & 0.25 & 0.25 & 0.25 & 0.25 & 0.25 & 0.23 & 0.23 & 0.22 & 0.23 & 0.19 & 0.21 \\
\hline $\mathrm{Ni}$ & 0.00 & 0.00 & 0.00 & 0.00 & 0.00 & 0.00 & 0.00 & 0.00 & 0.00 & 0.00 & 0.00 \\
\hline $\mathrm{Mn}$ & 0.01 & 0.01 & 0.01 & 0.01 & 0.01 & 0.01 & 0.01 & 0.01 & 0.01 & 0.01 & 0.01 \\
\hline $\mathrm{Fe}^{3+}$ & 0.07 & 0.09 & 0.10 & 0.09 & 0.09 & 0.09 & 0.09 & 0.08 & 0.08 & 0.08 & 0.10 \\
\hline Total & 4.00 & 4.00 & 4.00 & 4.00 & 4.00 & 4.00 & 4.00 & 4.00 & 4.00 & 4.00 & 4.00 \\
\hline $\mathrm{Mg} \#$ & 0.77 & 0.77 & 0.77 & 0.77 & 0.77 & 0.78 & 0.79 & 0.79 & 0.78 & 0.81 & 0.80 \\
\hline
\end{tabular}

Notes: $\mathrm{Fe}^{3+}$ calculated from charge balance; $\mathrm{Mg} \#=\mathrm{Mg} /(\mathrm{Mg}+\mathrm{Fe})$.

asmuch as the majority of our samples have compressional-wave velocities below values predicted based on modal content or normative constituents, there must be higher-order controls on velocity behavior. Static greenschist facies metamorphism characterizes the alteration of all the samples examined. In Table 5, we have compiled intensity of alteration data based on petrographic analyses of polished thin sections. Plagioclase is the least altered of the major mineral phases, but it is ubiquitously partially altered to albitic plagioclase. These grains are also characterized by a markedly dusty appearance due to minute grains of clay minerals. Pyroxene crystals, on the other hand, range from $75 \%$ fresh to completely altered (Table 5) to fibrous mats of tremolite-actinolite. Figure 6 demonstrates that there is a moderate correlation between increasing total alteration in the gabbroic rocks sampled and decreasing $V_{p}$. If we examine, however, the 
Table 4. Average plagioclase compositions from gabbroic rocks sampled at Site 894.

\begin{tabular}{|c|c|c|c|c|c|c|c|c|c|c|c|c|c|}
\hline Hole: & $894 \mathrm{~F}$ & $894 G$ & $894 \mathrm{G}$ & $894 \mathrm{G}$ & $894 \mathrm{G}$ & $894 \mathrm{G}$ & $894 \mathrm{G}$ & $894 \mathrm{G}$ & $894 \mathrm{G}$ & $894 \mathrm{G}$ & $894 \mathrm{G}$ & $894 \mathrm{G}$ & $894 \mathrm{G}$ \\
\hline Core, section: & $2 \mathrm{R}-1$ & $4 \mathrm{R}-1$ & $6 \mathrm{R}-2$ & $7 R-1$ & $8 R-2$ & 10R-1 & $11 R-1$ & $13 R-3$ & $15 \mathrm{R}-1$ & $18 \mathrm{R}-1$ & $19 \mathrm{R}-1$ & 20R-1 & $20 \mathrm{R}-1$ \\
\hline Interval: & $21-23$ & $91-93$ & $82-85$ & $43-45$ & $107-109$ & $85-87$ & $135-137$ & $13-15$ & $77-80$ & $130-132$ & $37-39$ & $19-22$ & $56-59$ \\
\hline Depth (m): & 9.53 & 45.91 & 57.05 & 65.25 & 71.08 & 79.95 & 85.47 & 106.38 & 119.57 & 132.22 & 140.89 & 145.82 & 146.19 \\
\hline \multicolumn{14}{|l|}{ Component (wt\%) } \\
\hline $\mathrm{Na}_{2} \mathrm{O}$ & 4.80 & 4.30 & 4.30 & 4.50 & 4.40 & 4.40 & 4.10 & 4.40 & 4.20 & 4.20 & 4.60 & 4.10 & 4.40 \\
\hline $\mathrm{SiO}_{2}$ & 54.00 & 53.00 & 52.00 & 53.00 & 53.00 & 52.00 & 52.00 & 52.00 & 52.00 & 52.00 & 53.00 & 52.00 & 52.00 \\
\hline $\mathrm{Al}_{2} \mathrm{O}_{3}$ & 28.70 & 29.60 & 29.50 & 29.10 & 29.40 & 29.40 & 29.90 & 29.30 & 29.60 & 29.80 & 29.20 & 30.00 & 29.40 \\
\hline $\mathrm{MgO}$ & 0.00 & 0.10 & 0.10 & 0.10 & 0.10 & 0.10 & 0.10 & 0.10 & 0.20 & 0.10 & 0.10 & 0.10 & 0.10 \\
\hline $\mathrm{SrO}$ & 0.00 & 0.00 & 0.00 & 0.00 & 0.00 & 0.00 & 0.00 & 0.00 & 0.00 & 0.00 & 0.00 & 0.00 & 0.00 \\
\hline $\mathrm{K}_{2} \mathrm{O}$ & 0.10 & 0.10 & 0.10 & 0.10 & 0.10 & 0.10 & 0.00 & 0.10 & 0.10 & 0.00 & 0.10 & 0.00 & 0.10 \\
\hline $\mathrm{CaO}$ & 12.00 & 13.00 & 13.00 & 12.00 & 13.00 & 13.00 & 13.00 & 13.00 & 13.00 & 13.00 & 12.00 & 13.00 & 13.00 \\
\hline $\mathrm{Fe}_{2} \mathrm{O}_{3}$ & 0.55 & 0.57 & 0.56 & 0.57 & 0.54 & 0.55 & 0.63 & 0.65 & 0.66 & 0.60 & 0.61 & 0.59 & 0.61 \\
\hline $\mathrm{BaO}$ & 0.00 & 0.00 & 0.00 & 0.10 & 0.10 & 0.00 & 0.00 & 0.00 & 0.00 & 0.00 & 0.10 & 0.00 & 0.00 \\
\hline Total & 99.89 & 100.00 & 99.27 & 99.39 & 99.70 & 99.25 & 99.49 & 99.17 & 99.20 & 100.00 & 100.00 & 100.00 & 99.16 \\
\hline \multicolumn{14}{|l|}{ Ions per 80} \\
\hline $\mathrm{Na}$ & 0.40 & 0.40 & 0.40 & 0.40 & 0.40 & 0.40 & 0.40 & 0.40 & 0.40 & 0.40 & 0.40 & 0.40 & 0.40 \\
\hline $\mathrm{Si}$ & 2.40 & 2.40 & 2.40 & 2.40 & 2.40 & 2.40 & 2.40 & 2.40 & 2.40 & 2.40 & 2.40 & 2.40 & 2.40 \\
\hline $\mathrm{Al}$ & 1.50 & 1.60 & 1.60 & 1.60 & 1.60 & 1.60 & 1.60 & 1.60 & 1.60 & 1.60 & 1.60 & 1.60 & 1.60 \\
\hline $\mathrm{Mg}$ & 0.00 & 0.00 & 0.00 & 0.00 & 0.00 & 0.00 & 0.00 & 0.00 & 0.00 & 0.00 & 0.00 & 0.00 & 0.00 \\
\hline $\mathrm{Sr}$ & 0.00 & 0.00 & 0.00 & 0.00 & 0.00 & 0.00 & 0.00 & 0.00 & 0.00 & 0.00 & 0.00 & 0.00 & 0.00 \\
\hline $\mathrm{K}$ & 0.00 & 0.00 & 0.00 & 0.00 & 0.00 & 0.00 & 0.00 & 0.00 & 0.00 & 0.00 & 0.00 & 0.00 & 0.00 \\
\hline $\mathrm{Ca}$ & 0.60 & 0.60 & 0.60 & 0.60 & 0.60 & 0.60 & 0.60 & 0.60 & 0.60 & 0.60 & 0.60 & 0.60 & 0.60 \\
\hline $\mathrm{Fe}$ & 0.00 & 0.00 & 0.00 & 0.00 & 0.00 & 0.00 & 0.00 & 0.00 & 0.00 & 0.00 & 0.00 & 0.00 & 0.00 \\
\hline $\mathrm{Ba}$ & 0.00 & 0.00 & 0.00 & 0.00 & 0.00 & 0.00 & 0.00 & 0.00 & 0.00 & 0.00 & 0.00 & 0.00 & 0.00 \\
\hline Total & 5.00 & 5.00 & 5.01 & 5.01 & 5,00 & 5.01 & 5.01 & 5.01 & 5.01 & 5.01 & 5.00 & 5.01 & 5.01 \\
\hline An & 0.58 & 0.62 & 0.62 & 0.60 & 0.61 & 0.61 & 0.63 & 0.61 & 0.62 & 0.63 & 0.59 & 0.64 & 0.61 \\
\hline
\end{tabular}

Note: $\mathrm{An}=$ Anorthite\# as $\mathrm{Ca} /(\mathrm{Ca}+\mathrm{Na}+\mathrm{K})$.

ratio of fresh pyroxene to the original modal pyroxene content plotted relative to $V_{p}$, we recognize a much more robust correlation (Fig. 7). More accurate quantification of the modal proportions of minerals in these samples is currently under way through enhanced image analysis. Considering that the effects of porosity variability have been minimized by velocity measurements at elevated pressure, density contrasts cannot fully account for $V_{p}$ variability (range of $1 \mathrm{~km} / \mathrm{s}$ at any given density, Fig. 2), and we can demonstrate no other systematic chemical or mineralogical trends, we surmise that the velocity behavior in the gabbroic rocks from near Hess Deep is primarily controlled by the relative degree of alteration of pyroxene. The reaction whereby pyroxene is consumed to produce amphibole:

$$
\begin{gathered}
3(\mathrm{Fe}, \mathrm{Mg}) \mathrm{SiO}_{3}+\underset{\text { crthopyroxene }}{2 \mathrm{Ca}(\mathrm{Fe}, \mathrm{Mg}) \mathrm{Si}_{2} \mathrm{O}_{6}}+\mathrm{SiO}_{2}+\mathrm{H}_{2} \mathrm{O} \\
\text { clinopyroxene quartz water } \\
=\mathrm{Ca}_{2}(\mathrm{Fe}, \mathrm{Mg})_{5} \mathrm{Si}_{8} \mathrm{O}_{22}(\mathrm{OH})_{2} \\
\text { actinolite }
\end{gathered}
$$

conserves ferromagnesian and calcic components and only involves the addition of minor water and $\mathrm{SiO}_{2}$; therefore, changes in bulk chemistry of the gabbros will be insignificant. At $200 \mathrm{MPa}$, however, the $V_{p}$ of actinolite is $5.8 \mathrm{~km} / \mathrm{s}$ (Fox et al., 1973) as opposed to 7.2 $\mathrm{km} / \mathrm{s}$ for pyroxene. In a rock that starts with $50 \%$ pyroxene and $50 \%$ plagioclase (gabbroic modal composition), for each $10 \%$ of the pyroxene converted to amphibole, we would expect $\sim 0.1 \mathrm{~km} / \mathrm{s}$ drop in velocity. This value is precisely reflected in the slope of the best-fit line in Figure 7. Alteration of pyroxene to amphibole also explains the discrepancy between MAW calculated from chemical analysis and that determined from velocity-density relations. Major element chemical composition is not significantly affected by this phase change; thus, MAW calculated from either the fresh rock or the altered rock will remain constant. The density of the altered rock is lowered radically, however, due to volumetric expansion. Therefore, data from these rocks in various stages of alteration, when plotted in velocity-density space (as in Fig. 1), will show a pronounced shift toward lower densities relative to their fresh equivalents.

We also recognize the potential that preferred mineral orientation may contribute to velocity behavior. If we examine data from mutually orthogonal pairs of minicores for various lithologies sampled on
Leg 147 (Fig. 8), for all rock types, the pairs have effectively the same density but differences of up to $0.4 \mathrm{~km} / \mathrm{s}$ in velocity. In this plot, offsets in $V_{p}$-density space between sample pairs parallel to the trend defined by all the data (dashed line) are due to sample heterogeneity. Offsets oblique to the overall trend are due to anisotropy effects, largely dictated by preferred mineral orientation. Iturrino et al. (this volume) suggest that preferred orientation of plagioclase is responsible for the slow velocities measured in orthogonal sample pairs from the gabbroic section. Tabular plagioclase induces lower velocities in a direction parallel to cleavage planes as compared to velocities measured perpendicular to cleavage. Since moderately dipping (average $75^{\circ}$ relative to core axis) magmatic foliation was commonly recognized in the gabbroic rocks from Site 894 (Gillis, Mével, Allan, et al., 1993), and because minicores were taken perpendicular and parallel to the core axis, maximum velocities for these samples would be at some oblique angle to the minicore axis. This type of preferred mineral orientation coupled to velocity decrease has been described for a much more extensive data set from Hole 735B, Southwest Indian Ridge (Iturrino et al., 1991).

Anomalously low seismic velocities, by as much as $1 \mathrm{~km} / \mathrm{s}$, are responsible for most of the difference between predicted MAW from density-velocity systematics and values calculated from chemical analyses for the serpentinized peridotites. For nearly all the peridotites examined in this investigation, the dominant vector of velocity difference between closely spaced orthogonal pairs of samples is oblique to the overall trend in velocity-density space exhibited by the entire suite of samples (Fig. 8). Nearly all of the peridotites sampled from Site 895 exhibit a strong, subhorizontal (relative to the core axis) foliation defined by the serpentine fabric (see Iturrino et al., this volume). In contrast to the gabbroic rocks, where no clear trend in orthogonal pairs data is evident, in each case for the peridotite samples, the core taken horizontally has a higher $V_{p}$ than its vertically oriented counterpart. However, mineral alignment is not the only control on velocity behavior for these rocks.

Figure 9 shows compressional-wave velocities and intensity of serpentinization for all the rocks sampled from Site 895 for this study. The distinct correlation $\left(\mathrm{R}^{2}=0.87\right)$ between $V_{p}$ and total alteration or degree of serpentinization indicates alteration is the primary control on velocity behavior in these serpentinized peridotites. To investigate whether this is a phenomenon unique to samples from near Hess 
Holes $894 \mathrm{~F}, \mathrm{G}$

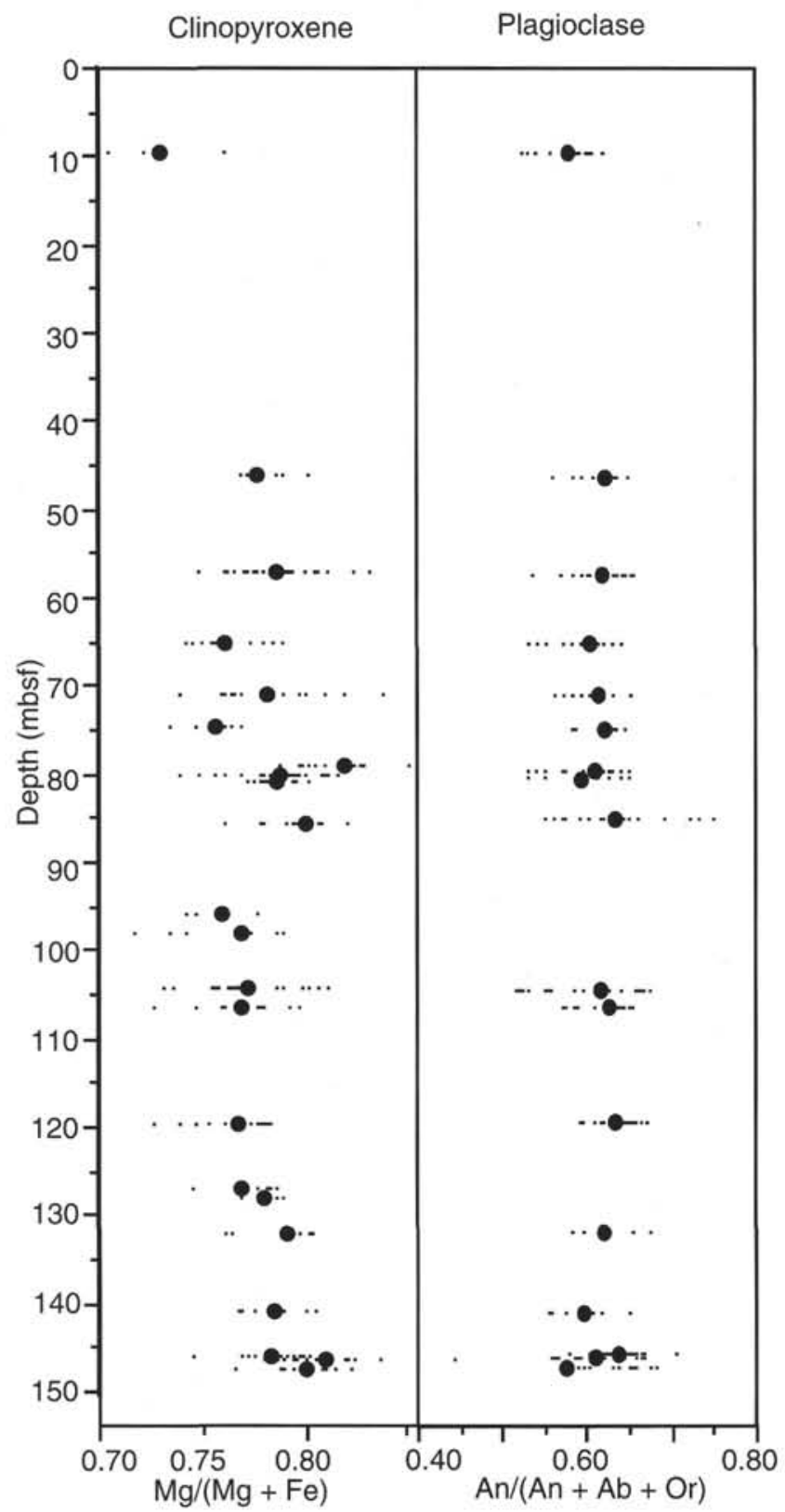

Figure 4. Clinopyroxene magnesium number and plagioclase anorthite content plotted as a function of depth of recovery for samples from Site 894. Small points are analyzed compositions, large solid circles are average compositions.

Deep, we have compiled from the literature compressional-wave velocity and degree of serpentinization data for peridotites from several environments (Fig. 10). When the data from Leg 147 are compiled with these, an even stronger correlation $\left(R^{2}=0.93\right)$ is evident.

Natland and Dick (this volume) argue that the oceanic crust near Hess Deep formed by crystallization in a vertical network of fractures that opened and closed repeatedly. Based on the lack of cyclic variations in chemical compositions of minerals, this model suggests that the sequence of isotropic and layered gabbros sampled at Site 894 did not crystallize from a stratiform intrusion. The complex variability in the velocity behavior of the gabbroic rocks from Hess Deep (Fig. 2) might be used to support this interpretation.
Based on local topography and drilling conditions, the scientific party on board the JOIDES Resolution speculated that Site 894 might have been located on a talus pile that lacked stratigraphic coherency. If, however, the increase in compressional-wave velocity with increase in depth in the samples from Site 894 and the correlation of that data with concomitant mineral chemistry profiles (Fig. 5) is real, then Hole $894 \mathrm{G}$ penetrated a coherent block of basement. The change in lithology coincident with the velocity spike may well represent a fresh input of magma into an existing chamber, which began precipitating a more primitive composition gabbronorite in response. One interesting result of this study is that without the break highlighted by the velocity profile as a marker horizon, the spike-and-decay pattern in the pyroxene chemistry profile becomes much less obvious. An alternative but less attractive explanation might be that drilling intercepted two large blocks, and the velocity spike and lithology change marks the contact between the two blocks. This would imply that the apparent spike-and-decay pattern in mineral chemistry profiles is serendipitous. Distinguishing between these scenarios can only be accomplished by future drilling in the gabbroic section at Hess Deep to establish lateral controls on geochemical and velocity variability.

Shallow cores from Site 895 span a range of compressional-wave velocities from below 5 to $7 \mathrm{~km} / \mathrm{s}$. This variability is lithology dependent (high $V_{p}$ for troctolite and olivine gabbro, lower $V_{p}$ for serpentinized peridotite) and dependent on degree of serpentinization. The distinct grouping of velocity data for each deeper core from Site 895 , without obvious stratigraphic control, suggests we may not have had sufficient penetration or recovery to identify an increasing velocity profile with depth. Alternatively, the expected velocitydepth relationship may have been completely overprinted by exhumation, emplacement, and hydration processes or, possibly, we penetrated a series of relatively small blocks (less than $10 \mathrm{~m}$ across, the length of a cored section) that have been randomly juxtaposed in a talus pile.

Finally, we can examine how the velocity data we have acquired from the gabbroic section at Hess Deep compare to the thick gabbro section sampled during ODP Leg 118. As illustrated in Figure 11, drilling at Hole $735 \mathrm{~B}$ recovered a thick section $(>500 \mathrm{~m})$ of gabbroic rock that exhibited a vague but identifiable increase in velocity with depth (data from Iturrino et al., 1991). The samples recovered from Hess Deep also have a distinct increase in velocity with depth (Fig. 3 ); however, the gradient is an order of magnitude greater than expected for normal oceanic crust and from the gradient observed in velocity data from Hole 735B. Inspection of the Hole 735B data reveals a slow increase in $V_{p}$ with depth accentuated by several sharp inflections where $V_{p}$ rapidly decreases at depths of $\sim 110 \mathrm{mbsf}, 240 \mathrm{mbsf}$, and 390 mbsf. Below each of these inflections the $V_{p}$ continues to steadily increase. In Figure 12, we have replotted the velocity depth profile from Hole $735 \mathrm{~B}$ next to a similar profile for Site 894 from Hess Deep. We have superimposed estimated best-fit lines to subsets of the data from Hole $735 \mathrm{~B}$ that terminate at the inflection points where there is a sharp decrease in $V_{p}$. Not only do these line segments appear to track the change in velocity with depth closely, the slopes of the individual line segments are very similar to the velocity gradient over the $150 \mathrm{~m}$ penetrated at Site 894 . We cannot at this time rule out the possibility that this correlation is completely random, particularly because penetration at Site 894 was limited to just over $150 \mathrm{~m}$; however, these data suggest that there may be a structural control, with a wavelength on the order of $150 \mathrm{~m}$, that is present in both sections of oceanic lower crustal gabbros that have been sampled to date. Once again we argue that only by drilling more and deeper holes at Hess Deep can we test the validity of this observation.

\section{SUMMARY}

This study combines data sets constraining the physical properties of lower crustal and upper mantle rocks collected from near the fast- 


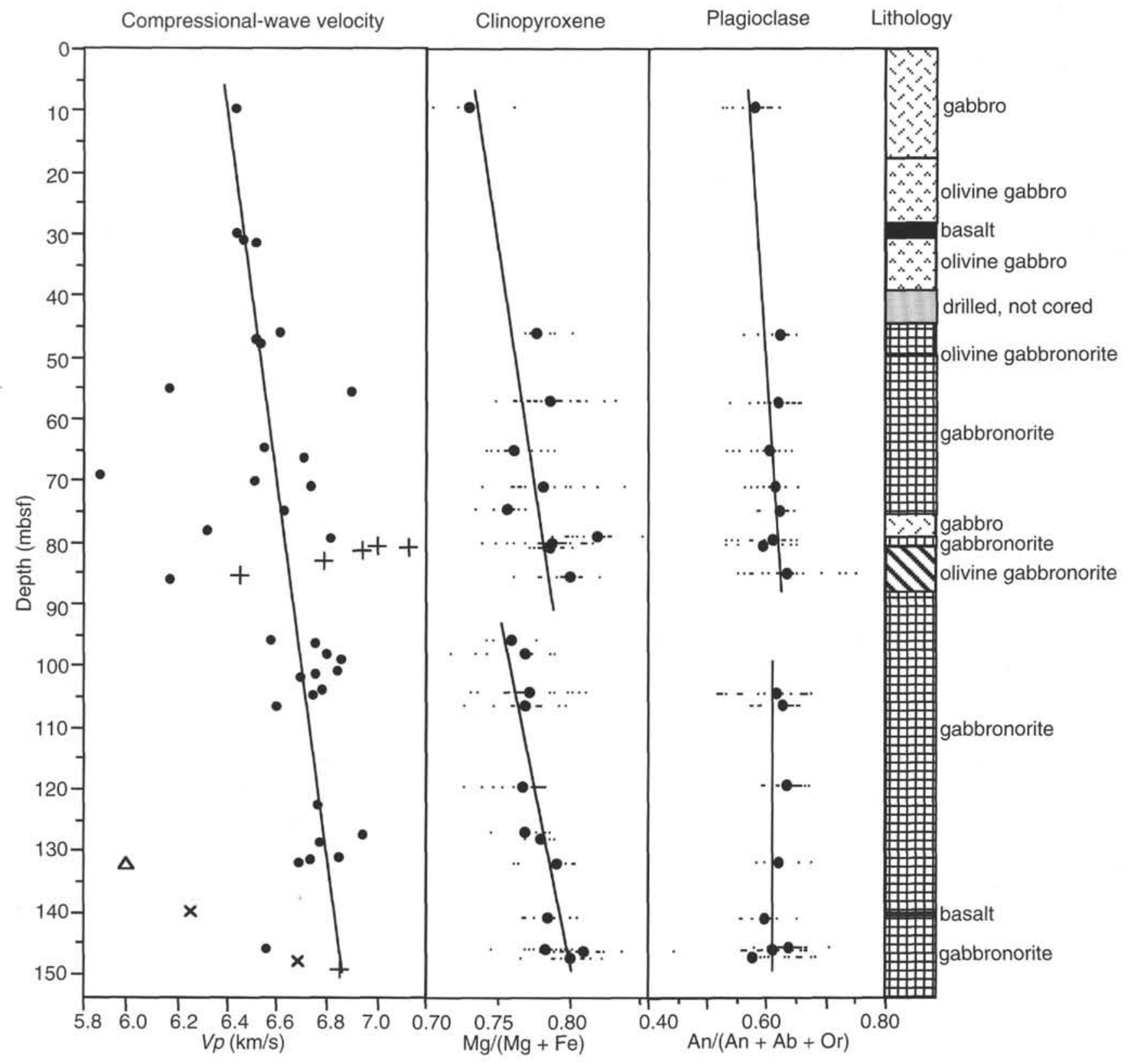

Figure 5. Compressional-wave velocity, clinopyroxene magnesium number, and plagioclase anorthite content plotted as a function of depth. Far right column is a schematic lithologic column for Site 894. The inflection in velocity data gradient between 80 and 90 mbsf is in response to a change in lithology. Superimposing the location of this inflection on mineral chemistry data highlights a potential spike-and-decay pattern, indicative of magma recharge.

spreading East Pacific Rise with geochemical and petrological data from the same sample suite. This multidisciplinary approach has generated interpretations that specifically address velocity models for the ocean crust.

1. No bulk-rock chemical indices exhibit apparent correlation with velocity variability.

2. Differences between mean atomic weight predicted from velocity-density systematics and calculated from bulk-rock geochemistry can be ascribed to alteration of pyroxene in the gabbroic rocks and to serpentinization and a strong serpentinite fabric in the peridotites.

3. The primary control on velocity behavior in the gabbroic rocks from Site 894 is alteration of pyroxene to amphibole. Preferred orientation of plagioclase in foliated gabbroic rocks can contribute to velocity behavior. In the serpentinized peridotites from Site 895 , the primary controls on velocity behavior are degree of serpentinization and velocity anisotropy dictated by a strong serpentinite fabric.

4. Comparison of velocity-depth profiles and mineral-composition profiles highlight compositional trends that are not readily apparent in compositional data alone. The consistent velocity gradient throughout the gabbroic section suggests a coherent block was drilled, rather than a talus pile. The lack of a similar trend in data from the ultramafic section indicates drilling at this site penetrated randomly juxtaposed small blocks, or that alteration has obscured any stratigraphically controlled velocity behavior.

5 . High velocity gradients over intervals of $\sim 150 \mathrm{~m}$ can be recognized in the data from Hole $735 \mathrm{~B}$, where we have sampled the most complete section of oceanic lower crust to date. These intervals are characterized by a slow increase in $V_{p}$ with depth terminated by a sud- 
den decrease in $V_{p}$ which offsets the intervals. Although drilling at Site 894 penetrated just over $150 \mathrm{~m}$, a velocity gradient of similar magnitude is present.

\section{ACKNOWLEDGMENTS}

This study was supported by grants from JOI-USSAC to DJM and GJI, and from the Office of Naval Research to NIC. This paper benefitted from constructive reviews from P.A. Berge and an anonymous reviewer.

\section{REFERENCES}

Birch, F., 1960. The velocity of compressional waves in rocks to 10 kilobars, 1. J. Geophys. Res., 65:1083-1102.

1961. The velocity of compressional waves in rocks to 10 kilobars, 2. J. Geophys. Res., 66:2199-2224.

Christensen, N.I., 1966. Elasticity of ultrabasic rocks. J. Geophys. Res. 71:5921-5931.

Fox, P.J., Schreiber, E., and Peterson, J.J., 1973. The geology of the oceanic crust: compressional wave velocities of oceanic rocks. J. Geophys. Res., $78: 5155-5172$.

Gillis, K., Mével, C., Allan, J., et al., 1993. Proc. ODP, Init. Repts., 147: College Station, TX (Ocean Drilling Program).
Hill, R., 1952. The elastic behavior of a crystalline aggregate. Proc. Phys, Soc. London, Sect. A, 65:349-354.

Iturrino, G.J., Christensen, N.I., Kirby, S., and Salisbury, M.H., 1991. Seismic velocities and elastic properties of oceanic gabbroic rocks from Hole 735B. In Von Herzen, R.P., Robinson, P.T., et al., Proc. ODP, Sci. Results, 118: College Station, TX (Ocean Drilling Program), 227-244.

Kern, H., and Tubia, J.M., 1993. Pressure and temperature dependence of Pand S-wave velocities, seismic anisotropy and density of sheared rock from the Sierra Alpujata (Ronda peridotites, Southern Spain). Earth Planet. Sci. Lett., 119:191-205.

Miller, D.J., and Christensen, N.I., 1994. Seismic signature and geochemistry of an island arc: a multidisciplinary study of the Kohistan accreted terrane, northern Pakistan. J. Geophys. Res., 99:11623-11642.

Ramana, Y.V., and Rao, M.V.M.S., 1974. Compressional velocities in ultramafic rocks of India at pressures to five kilobars. Geophys. J. R. Astron. Soc., 37:207-212.

Thornton, C.P., and Tuttle, O.F., 1960. Chemistry of igneous rocks, Part I: differentiation index. Am. J. Sci., 258:664-684.

Date of initial receipt: 20 January 1995

Date of acceptance: 17 May 1995

Ms 147SR-028

Table 5. Intensity of alteration for gabbroic samples from Site 894.

\begin{tabular}{|c|c|c|c|c|c|c|c|}
\hline \multirow{2}{*}{$\begin{array}{l}\text { Core, section, } \\
\text { interval }(\mathrm{cm})\end{array}$} & \multicolumn{2}{|c|}{ Plagioclase (\%) } & \multicolumn{2}{|c|}{ Clinopyroxene (\%) } & \multicolumn{2}{|c|}{ Orthopyroxene (\%) } & \multirow{2}{*}{$\begin{array}{c}\text { Total } \\
\text { alteration }(\%)\end{array}$} \\
\hline & Fresh & Altered & Fresh & Altered & Fresh & Altered & \\
\hline $\begin{array}{l}147-894 \mathrm{~F}- \\
2 \mathrm{R}-1,21-23\end{array}$ & 46 & 5 & 12 & 35 & 0 & 0 & 40 \\
\hline $\begin{array}{l}147-894 \mathrm{G}- \\
4 \mathrm{R}-1,91-93 \\
6 \mathrm{R}-2,78-80 \\
7 \mathrm{R}-1,43-45 \\
8 \mathrm{R}-2,107-109 \\
9 \mathrm{R}-1,37-39 \\
9 \mathrm{R}-4,54-59 \\
9 \mathrm{R}-4,123-127 \\
10 \mathrm{R}-2,38-40 \\
11 \mathrm{R}-1,135-139 \\
11 \mathrm{R}-2,45-47 \\
12 \mathrm{R}-2,46-50 \\
12 \mathrm{R}-5,108-111 \\
13 \mathrm{R}-1,86-89 \\
13 \mathrm{R}-1,89-91 \\
13 \mathrm{R}-3,13-15 \\
17 \mathrm{R}-2,87-89 \\
18 \mathrm{R}-1,72-76 \\
18 \mathrm{R}-1,130-134 \\
\text { 19R-1, 37-39 } \\
20 \mathrm{R}-1,60-62 \\
20 \mathrm{R}-2,33-37\end{array}$ & $\begin{array}{l}55 \\
40 \\
50 \\
45 \\
55 \\
50 \\
50 \\
50 \\
40 \\
36 \\
55 \\
40 \\
43 \\
43 \\
53 \\
55 \\
55 \\
55 \\
50 \\
43 \\
48\end{array}$ & $\begin{array}{r}0 \\
5 \\
10 \\
10 \\
5 \\
5 \\
5 \\
5 \\
10 \\
14 \\
5 \\
5 \\
10 \\
10 \\
2 \\
5 \\
5 \\
5 \\
10 \\
2 \\
5\end{array}$ & $\begin{array}{r}15 \\
30 \\
10 \\
25 \\
10 \\
10 \\
20 \\
18 \\
10 \\
2 \\
10 \\
10 \\
15 \\
20 \\
20 \\
15 \\
25 \\
25 \\
5 \\
10 \\
15\end{array}$ & $\begin{array}{l}15 \\
10 \\
10 \\
10 \\
20 \\
25 \\
10 \\
20 \\
30 \\
35 \\
10 \\
20 \\
15 \\
10 \\
16 \\
10 \\
10 \\
10 \\
25 \\
15 \\
20\end{array}$ & $\begin{array}{r}10 \\
10 \\
5 \\
5 \\
5 \\
2 \\
3 \\
2 \\
3 \\
3 \\
10 \\
5 \\
7 \\
7 \\
14 \\
10 \\
0 \\
0 \\
5 \\
10 \\
3\end{array}$ & $\begin{array}{r}5 \\
5 \\
10 \\
5 \\
5 \\
3 \\
5 \\
3 \\
7 \\
7 \\
10 \\
10 \\
8 \\
8 \\
5 \\
5 \\
5 \\
5 \\
10 \\
20 \\
7\end{array}$ & $\begin{array}{l}20 \\
20 \\
30 \\
25 \\
30 \\
36^{*} \\
23^{*} \\
28 \\
47 \\
54 \\
25 \\
35 \\
33 \\
28 \\
22 \\
20 \\
20 \\
20 \\
45 \\
37 \\
32\end{array}$ \\
\hline
\end{tabular}

Note: ${ }^{*}=$ includes altered olivine. 


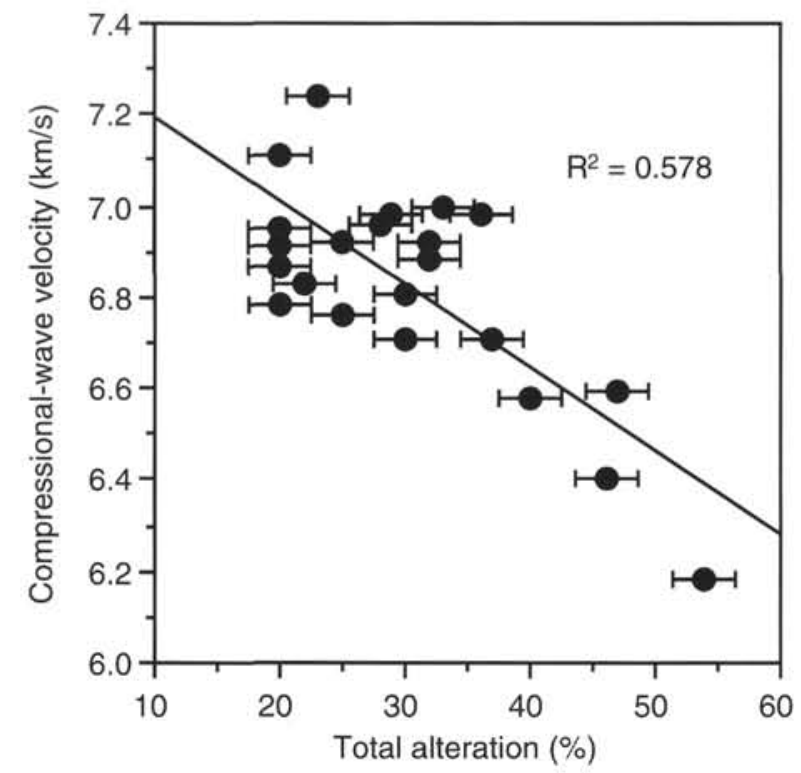

Figure 6. Total alteration plotted vs. $V_{p}$ for gabbroic rocks sampled from Site 894. Degree of alteration based on visual estimate with $5 \%$ margin of error.

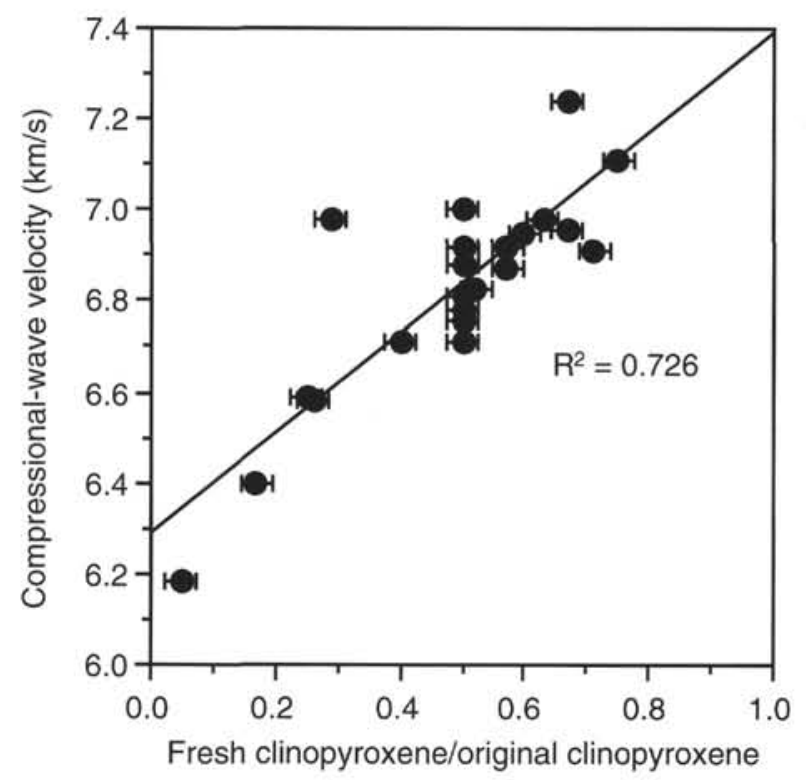

Figure 7. Ratio of fresh pyroxene to original modal pyroxene content plotted vs. $V_{p}$ for gabbroic rocks sampled from Site 894 . The distinct positive correlation and the lack of contributions to velocity behavior from other possible controls indicate alteration of pyroxene is the primary control on $V_{p}$ for these samples. Note that recalculation of best-fit line excluding the two points that fall well above the observed data trend (olivine-bearing gabbros) yields $R^{2}=$ 0.88. Same error margin as for Figure 6.

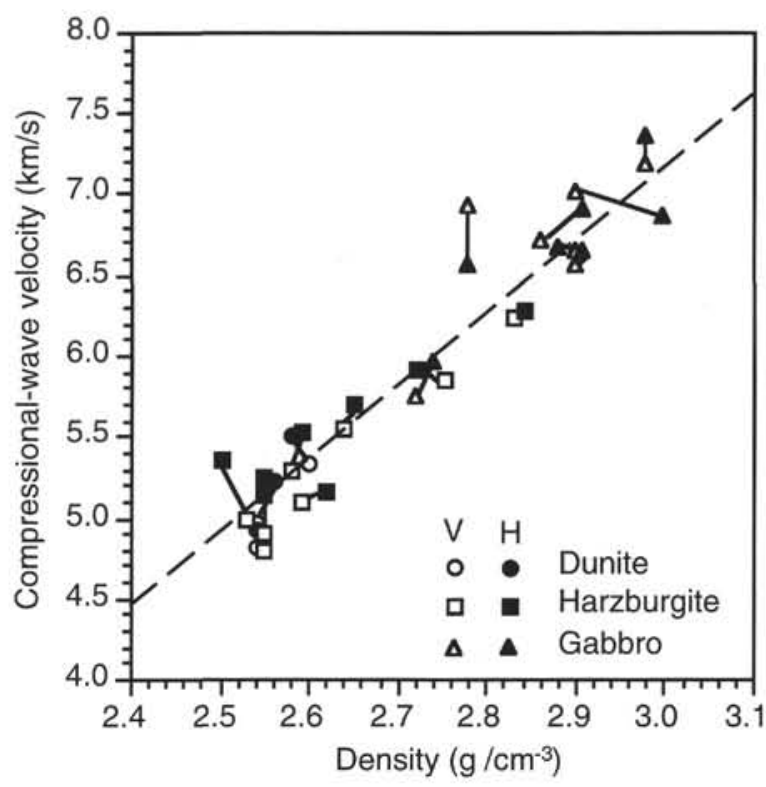

Figure 8. $V_{p}$ vs. density data for orthogonal pairs of samples collected during Leg 147 . For most samples, densities are very similar, but velocities vary by as much as $0.4 \mathrm{~km} / \mathrm{s}$. Open symbols indicate minicores sampled parallel to core axis (vertical). Solid symbols indicate minicores sampled perpendicular to core axis (horizontal).

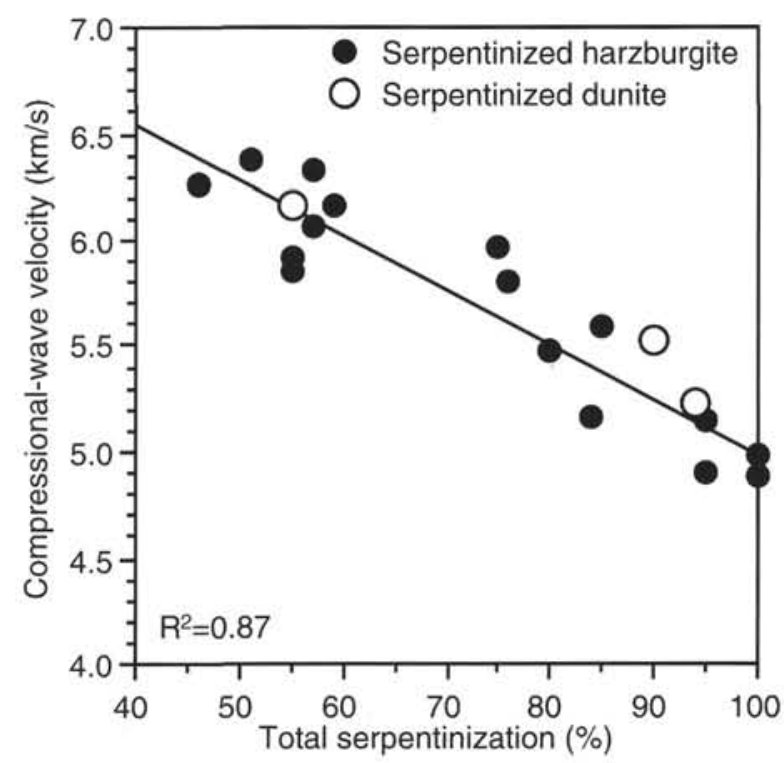

Figure 9. Correlation between $V_{p}$ and intensity of serpentinization for ultramafic rocks sampled at Site 895 . 


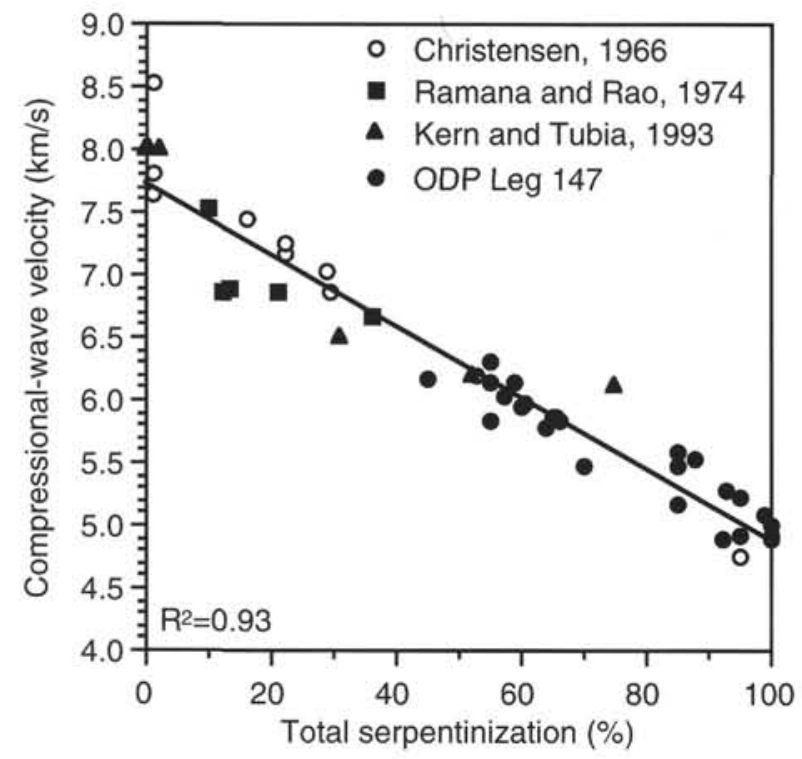

Figure 10. $V_{p}$ relationship to total intensity of serpentinization for various serpentinized peridotites worldwide. Data from Christensen (1966) are from an ultramafic body in the Santa Lucia Range, California. Ramana and Rao (1974) data are from variably serpentinized ultramafic rocks from India. Kern and Tubia (1993) data are from serpentinized peridotites in Spain. Data from ultramafic rocks recovered from near Hess Deep superimpose on the high intensity end of the serpentinization trend of data from serpentinized peridotites worldwide.

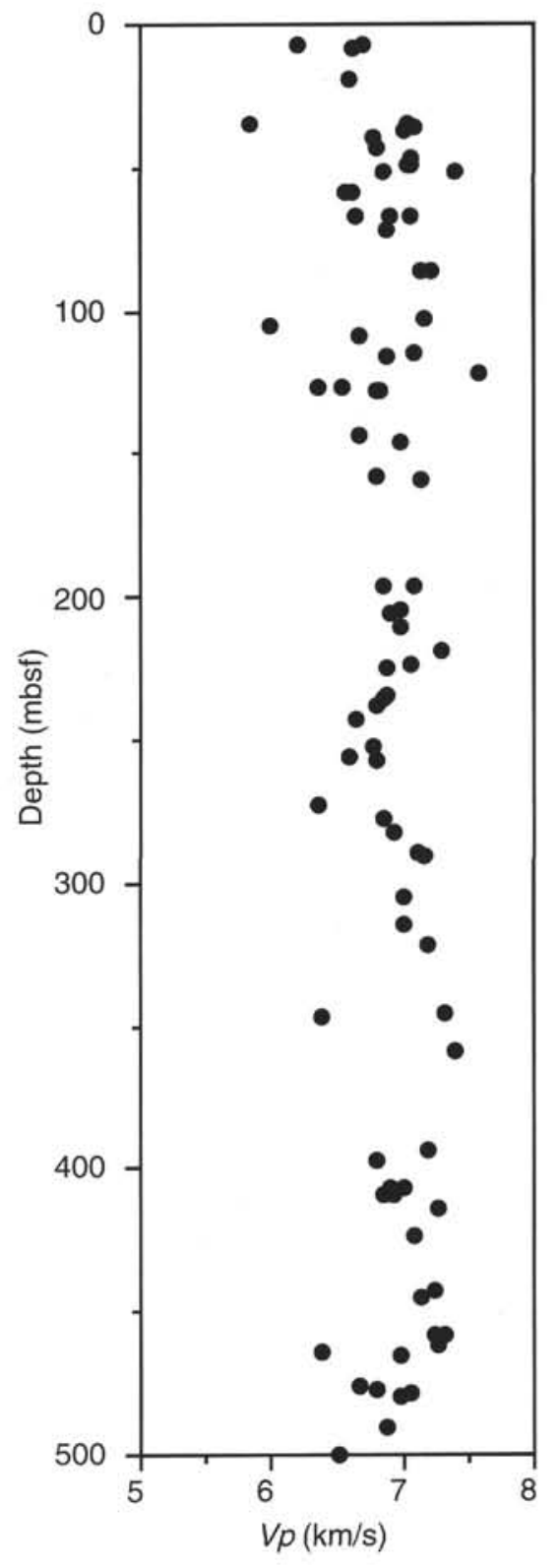

Figure 11. Laboratory-measured compressional-wave velocity at $200 \mathrm{MPa}$ plotted as a function of depth for gabbroic rocks from Hole 735B, Southwest Indian Ridge. Data from Iturrino et al. (1991). 


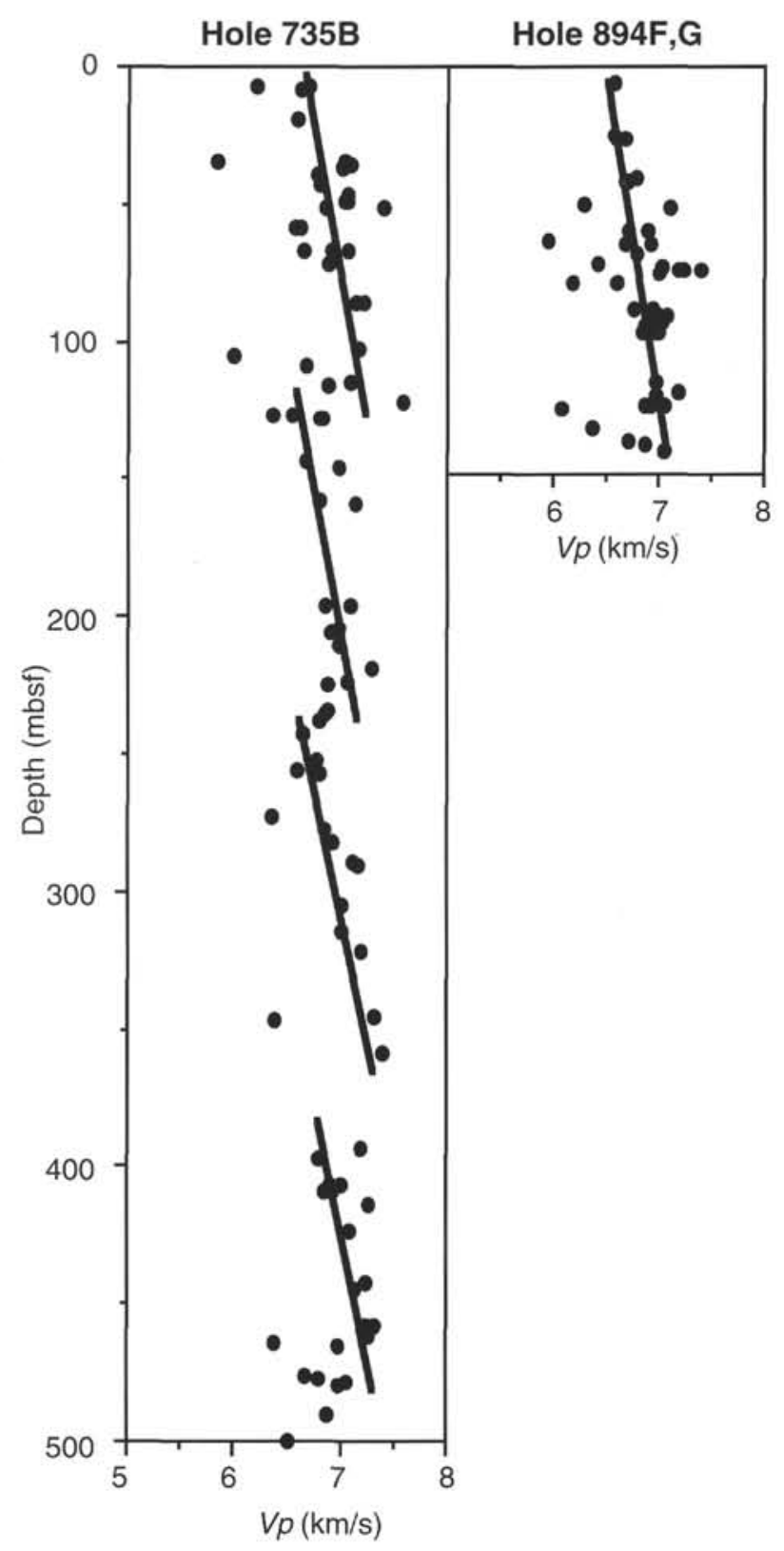

Figure 12. Laboratory-measured compressional-wave velocity at $200 \mathrm{MPa}$ plotted as a function of depth for samples from Hole $735 \mathrm{~B}$ (Southwest Indian RidgeSWIR) and Holes 894F and 894G (Hess Deep). Lines are estimated best-fit trends to data for illustrative purposes. 